\title{
Abiotic formation of $\mathrm{O}_{2}$ and $\mathrm{O}_{3}$ in high- $\mathrm{CO}_{2}$ terrestrial atmospheres
}

\author{
A. Segura ${ }^{1,5, \star}$, V. S. Meadows ${ }^{1,5}$, J. F. Kasting ${ }^{2,5}$, D. Crisp ${ }^{3,5}$, and M. Cohen ${ }^{4,5}$ \\ 1 California Institute of Technology, 1200 East California Blvd. Mail stop 220-6, Pasadena CA, 91125 USA \\ e-mail: antigona@nucleares.unam.mx \\ 2 Pennsylvania State University, 443 Deike Bldg. State College, PA 16802, USA \\ 3 NASA Jet Propulsion Laboratory, 4800 Oak Grove Dr. Pasadena, CA 91109, USA \\ 4 University of California, Radio Astronomy Laboratory, 601 Campbell Hall, Berkeley, CA 94720 USA \\ 5 Members of the Virtual Planetary Laboratory, a project of the NASA Astrobiology Institute
}

Received 30 October 2006 / Accepted 28 June 2007

\section{ABSTRACT}

\begin{abstract}
Context. Previous research has indicated that high amounts of ozone $\left(\mathrm{O}_{3}\right)$ and oxygen $\left(\mathrm{O}_{2}\right)$ may be produced abiotically in atmospheres with high concentrations of $\mathrm{CO}_{2}$. The abiotic production of these two gases, which are also characteristic of photosynthetic life processes, could pose a potential "false-positive" for remote-sensing detection of life on planets around other stars. We show here that such false positives are unlikely on any planet that possesses abundant liquid water, as rainout of oxidized species onto a reduced planetary surface should ensure that atmospheric $\mathrm{H}_{2}$ concentrations remain relatively high, and that $\mathrm{O}_{2}$ and $\mathrm{O}_{3}$ remain low. Aims. Our gool is to determine the amount of $\mathrm{O}_{3}$ and $\mathrm{O}_{2}$ formed in a high $\mathrm{CO}_{2}$ atmosphere for a habitable planet without life. Methods. We use a photochemical model that considers hydrogen $\left(\mathrm{H}_{2}\right)$ escape and a detailed hydrogen balance to calculate the $\mathrm{O}_{2}$ and $\mathrm{O}_{3}$ formed on planets with 0.2 of $\mathrm{CO}_{2}$ around the Sun, and 0.02, 0.2 and 2 bars of $\mathrm{CO}_{2}$ around a young Sun-like star with higher UV radiation. The concentrations obtained by the photochemical model were used as input in a radiative transfer model that calculated the spectra of the modeled planets.

Results. The $\mathrm{O}_{3}$ and $\mathrm{O}_{2}$ concentrations in the simulated planets are extremely small, and unlikely to produce a detectable signature in the spectra of those planets.

Conclusions. With a balanced hydrogen budget, and for planets with an active hydrological cycle, abiotic formation of $\mathrm{O}_{2}$ and $\mathrm{O}_{3}$ is unlikely to create a possible false positive for life detection in either the visible/near-infrared or mid-infrared wavelength regimes.
\end{abstract}

Key words. astrobiology - infrared: general - ultraviolet: stars

\section{Introduction}

Most of the $\mathrm{O}_{2}$ in Earth's present atmosphere is thought to have been produced by oxygenic photosynthesis, followed by burial of organic carbon in marine sediments (Cloud 1972; Walker 1977; Holland 1978, 1984, 2002). Prior to the origin of life, and of $\mathrm{O}_{2}$-producing life in particular, atmospheric $\mathrm{O}_{2}$ mixing ratios are thought to have been very low, $10^{-13}$ by volume, or $\sim 10^{-12}$ PAL at the surface (Walker 1977; Kasting et al. 1979; Kasting 1993; Kasting \& Catling 2003) Here, "PAL" means "times the Present Atmospheric Level", which is 21 percent by volume, or 0.21 bars. $\mathrm{O}_{2}$ concentrations in the upper atmosphere of the early Earth could have been much higher, up to $\sim 10^{-3}$ by volume (Kasting \& Catling 2003), as a consequence of photolysis of $\mathrm{CO}_{2}$, followed by recombination of $\mathrm{O}$ atoms to make $\mathrm{O}_{2}$. A small amount of ozone, $\mathrm{O}_{3}$, could have been formed from this $\mathrm{O}_{2}$, but not enough to provide an effective shield against solar UV radiation (Levine et al. 1979; Kasting \& Donahue 1980; Kasting 1993, and Refs. therein; Cockell \& Horneck 2001). Early life therefore probably evolved in an anoxic, high-UV environment.

While the question of abiotic $\mathrm{O}_{2}$ and $\mathrm{O}_{3}$ levels has historically been of interest to geologists and biologists, it has recently become an important issue for astronomers as well.

^ Present address: Instituto de Ciencias Nucleares, Universidad Nacional Autónoma de México, Circuito Exterior, CU Apartado Postal 70-543, 04510, México, D.F.
Within the next 15-20 years, space-based telescopes, such as the two Terrestrial Planet Finder (TPF) missions planned by NASA (http://planetquest.jpl.nasa.gov/TPF/tpf_c_ team.cfm) and ESA's Darwin mission (http://ast.star. rl.ac.uk/darwin/), will hopefully search for Earth-sized planets around other stars and attempt to obtain spectra of their atmospheres and surfaces. TPF-C (a visible/near-IR coronagraph) will be sensitive to the $0.76-\mu \mathrm{m}$ band of $\mathrm{O}_{2}$, while TPF-I and Darwin (both thermal-IR interferometers) will be sensitive to the $9.6-\mu \mathrm{m}$ band of $\mathrm{O}_{3}$. Both of these gases are considered to be possible biomarker compounds (Owen 1980; Angel et al. 1986; Leger et al. 1993). The question as to whether they can be produced abiotically is therefore of great potential relevance to the interpretation of data from these future missions.

Situations in which either $\mathrm{O}_{2}$ or $\mathrm{O}_{3}$, or both, might accumulate abiotically have been identified by a number of different authors. Some of these situations are legitimate "false positives" for life, while others might be misleading. Two situations, in particular, appear capable of producing "false positive" signals. The first is a runaway greenhouse planet, like early Venus, on which large amounts of hydrogen escape from a hot, moist atmosphere (Kasting 1997; Schindler \& Kasting 2000). Because the hydrogen originates from $\mathrm{H}_{2} \mathrm{O}$, oxygen is left behind. The escape of a terrestrial ocean equivalent of hydrogen, unaccompanied by oxygen sinks, could leave an atmosphere containing $\sim 240$ bars of $\mathrm{O}_{2}$ (Kasting 1997). A second conceivable "false positive" is a frozen, Mars-like planet that is large enough to 
retain heavy gases but too small to maintain volcanic outgassing (Kasting 1997; Schindler \& Kasting 2000). The frozen surface would inhibit the loss of oxygen by reaction with reduced minerals, whereas the lack of outgassing would eliminate reaction with reduced volcanic gases (primarily $\mathrm{H}_{2}$ ) as an oxygen sink. The martian atmosphere contains $0.1 \% \mathrm{O}_{2}$ and would likely have even more if the planet were slightly larger so that it did not lose oxygen to space by nonthermal loss mechanisms (McElroy \& Donahue 1972).

Both of the "false positives" mentioned above apply to planets that lie outside of the liquid water habitable zone around their parent star. The boundaries of this zone can be estimated to first order from climate models (Kasting et al. 1993), and it should be possible to determine observationally whether the planet's distance from its parent star falls outside these limits. Such a determination would not be definitive, as the theoretical limits of the habitable zone are uncertain, mostly because of the difficulty in simulating clouds. (The Kasting et al. 1993 model effectively puts the cloud layer at the planet's surface, thereby ignoring all cloud feedbacks.) Planets outside the habitable zone may also be distinguishable by the absence, or near absence, of gaseous $\mathrm{H}_{2} \mathrm{O}$ in their spectra, although water-rich planets near the inner edge might not obey this rule, and one should remain suspicious of $\mathrm{O}_{2}$ signals on such bodies. Still, the most important false positive issue is whether or not planets within the habitable zone could build up $\mathrm{O}_{2}$ or $\mathrm{O}_{3}$ abiotically.

Calculations predicting high, or relatively high, abiotic $\mathrm{O}_{2}$ and $\mathrm{O}_{3}$ concentrations have appeared sporadically in the literature over the past 40 years. All authors have realized that photolysis of $\mathrm{H}_{2} \mathrm{O}$, followed by escape of hydrogen to space, is a net source of oxygen. Berkner \& Marshall (1964, 1965, 1966, 1967) estimated abiotic $\mathrm{O}_{2}$ concentrations of $10^{-4}-10^{-3}$ PAL based on how much $\mathrm{O}_{2}$ buildup was needed to block out the UV radiation that dissociates $\mathrm{H}_{2} \mathrm{O}$. This is probably too little $\mathrm{O}_{2}$ to be detectable spectroscopically by a telescope like TPF-C; however, the associated ozone layer could conceivably be detected by a telescope like TPF-I or Darwin (Segura et al. 2003). In a study a few years later, Brinkman (1969) found abiotic $\mathrm{O}_{2}$ concentrations of up to 0.27 PAL. If correct, such an atmosphere would produce an absorption feature at $0.76 \mu \mathrm{m}$ about half as strong as that of Earth (Des Marais et al. 2002). Brinkmann obtained this high value because he assumed that precisely one-tenth of the $\mathrm{H}$ atoms produced by $\mathrm{H}_{2} \mathrm{O}$ photolysis escaped - a fraction that we now know is much too high.

All of these early studies were performed before the factors controlling hydrogen escape from Earth's atmosphere were well understood. Hunten (1973) showed that the H escape rate from Earth (and from Saturn's moon, Titan) is limited by the rate at which hydrogen can diffuse upwards through the homopause. (The homopause, near $90 \mathrm{~km}$ in Earth's atmosphere, is the altitude above which light gases begin to separate out from heavy gases. Equivalently, it marks the transition from turbulent vertical transport to molecular diffusion.) The resulting escape rate is termed the "diffusion-limited flux". Hydrogen can escape more slowly than the diffusion limit because of energy limitations higher up (see, e.g., Tian et al. 2005), but it cannot escape more rapidly. Walker (1977) applied this concept to the early Earth and showed that it implies extremely low ground-level prebiotic $\mathrm{O}_{2}$ concentrations, of the order of $10^{-13}$ PAL. This same reasoning applies to vertically resolved atmospheric photochemical models (Kasting et al. 1979; Kasting 1993; Kasting \& Catling 2003), although the $\mathrm{O}_{2}$ concentration in the stratosphere can be much higher $\left(10^{-3}\right)$, as mentioned earlier. High stratospheric $\mathrm{O}_{2}$ concentrations are favored by high $\mathrm{CO}_{2}$ levels and high solar UV fluxes (Canuto et al. 1982, 1983). It should be pointed out that the extremly high UV fluxes studied by Canuto et al. are unlikely to apply to the early Earth or to any analogous planet, as the $\mathrm{T}$ Tauri phase of stellar evolution was probably over long before the Earth was fully formed.

Although most recent models predict low abiotic $\mathrm{O}_{2}$ concentrations for planets within the habitable zone, contradictory results also appear. This happens, for example, in some calculations performed by Selsis et al. (2002) for a variety of Earth-like and Mars-like planets. The Earth-like planets all had significant volcanic sinks for oxygen, and so none of these cases produced high $\mathrm{O}_{2}$ or $\mathrm{O}_{3}$. However, their "early Mars-type" planet (Case B2), which assumed zero volcanic outgassing, exhibited 0.1 PAL of $\mathrm{O}_{2}$ and a "super" ozone layer with a column depth of $\sim 0.7 \mathrm{~atm} \mathrm{~cm}-$ roughly twice that of modern Earth. This model simulated a warm, humid, 1-bar $\mathrm{CO}_{2}$ atmosphere underlain by an ocean. If this calculation were valid, then such a planet would represent another possible "false positive" for life. Selsis et al. argued that the $\mathrm{O}_{3} 9.6-\mu \mathrm{m}$ band would be obscured by the neighboring 9.4- and 10.4- $\mu \mathrm{m}$ hot bands of $\mathrm{CO}_{2}$, and thus would not constitute a false positive for Darwin (or TPF-I). However, the $0.76-\mu \mathrm{m}$ band of $\mathrm{O}_{2}$ would be quite prominent in this atmosphere, and so it would remain an issue for TPF-C.

We argue here that the Selsis et al. calculation is incorrect, or at least over-simplified, and that this case is not a likely "false positive". More precisely, we suggest that abiotic $\mathrm{O}_{2}$ buildup is only likely to occur on dry or frozen planets, and such planets could be differentiated from warmer Earth-like planets by examining their spectra. The reason that $\mathrm{O}_{2}$ reached such high levels in the Selsis et al. calculation is that the authors failed to consider the effect of rainout of oxidized (or reduced) species on the atmospheric hydrogen budget. Photochemical production of oxidized species such as hydrogen peroxide, $\mathrm{H}_{2} \mathrm{O}_{2}$, followed by their reaction with reduced species in the crust or in seawater, should generate a net source of $\mathrm{H}_{2}$ (Kasting et al. 1984a; Kasting \& Brown 1998; Kasting \& Catling 2003). This $\mathrm{H}_{2}$ source (and $\mathrm{O}_{2}$ sink) was left out of the Selsis et al. model, allowing for a much higher abiotic $\mathrm{O}_{2}$ concentration. The next section describes the atmospheric hydrogen budget, along with the rest of our photochemical model, in more detail. We then use our model to simulate various Earth-like planets, with and without volcanic outgassing, and under high and low stellar UV flux, and we calculate abiotic $\mathrm{O}_{2}$ and $\mathrm{O}_{3}$ concentrations and accompanying visible and thermal-IR spectra. Our goal is to determine whether any additional "false positives" for $\mathrm{O}_{2}$ producing life could exist in high- $\mathrm{CO}_{2}$ atmospheres.

\section{Model description}

\subsection{The photochemical model}

The 1D photochemical model used in our study was developed for high- $\mathrm{CO}_{2} /$ high- $\mathrm{CH}_{4}$ terrestrial atmospheres by Pavlov et al. (2001) and was subsequently modified by Kharecha et al. (2005). The model simulates an anoxic atmosphere composed of 0.8 bar of $\mathrm{N}_{2}$ and variable amounts of $\mathrm{CO}_{2}$. We did not simulate a pure- $\mathrm{CO}_{2}$, "Mars-like" atmosphere explicitly; however, such an atmosphere is similar in principle to our high- $\mathrm{CO}_{2}$ cases. Our photochemical model contained 73 chemical species involved in 359 reactions and spanned the region from the planetary surface up to $64 \mathrm{~km}$ in 1-km steps. The solar zenith angle was fixed at $50^{\circ}$, and a two-stream approach was used for the radiative transfer. The continuity equation was solved at each height for each of the long-lived species, including transport by eddy 
a)

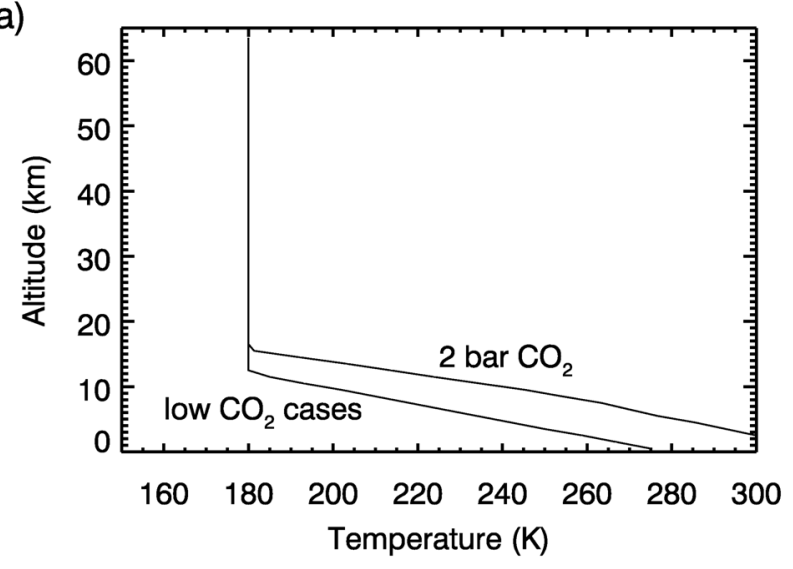

b)

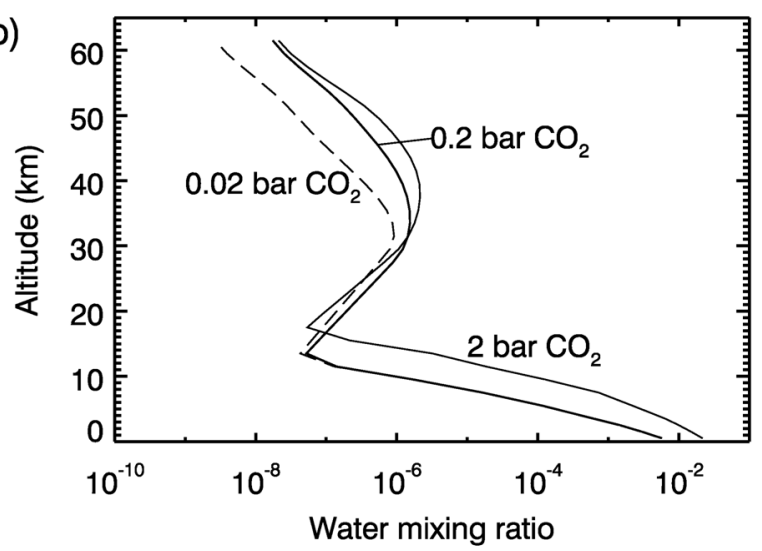

Fig. 1. a) Temperature profiles for the low- $\mathrm{CO}_{2}$ atmospheres $(0.2$ and 0.02 bars of $\left.\mathrm{CO}_{2}\right)$ and the high $\mathrm{CO}_{2}$ planet $\left(2\right.$ bars of $\mathrm{CO}_{2}$. b) Water profiles for the simulated planetary atmospheres around EK Dra.

and molecular diffusion. The combined equations were cast in centered finite difference form. Boundary conditions for each species were applied at the top and botton of the model atmosphere, and the resulting set of coupled differential equations was integrated to steady state using the reverse Euler method. Two different stellar UV fluxes were used, as described below. The eddy diffusion profile for most cases was assumed to be the same as the one measured on present Earth (Massie \& Hunten 1981), with modifications for atmospheres in which the total surface pressure exceeded one bar. All of the simulated planets were assumed to be devoid of life; hence, none of the compounds in the atmosphere was considered to have a biological source.

As a reference, or "standard", case we assumed a 1-bar, cloudless, "early-Earth" type atmosphere with 0.2 bar of $\mathrm{CO}_{2}$. This is roughly the amount of $\mathrm{CO}_{2}$ needed to compensate for a $30 \%$ reduction in solar luminosity, appropriate for a time near 4.5 Gyr ago (Kasting 1993). We did not perform coupled climate model calculations for this case; rather, we simply assumed that the temperature decreased with height from $278 \mathrm{~K}$ at the surface to $180 \mathrm{~K}$ at $12.5 \mathrm{~km}$, following a moist adiabat. Above that height, the atmosphere was assumed to be isothermal (Fig. 1). This is roughly consistent with the predictions of climate models (Kasting 1990). It is difficult for us to improve significantly on this assumption, as our own climate model (Pavlov et al. 2000) is not particularly accurate up in the Doppler-broadened stratosphere and mesosphere. The assumed solar UV flux in this model was the same as for Earth today. We also performed

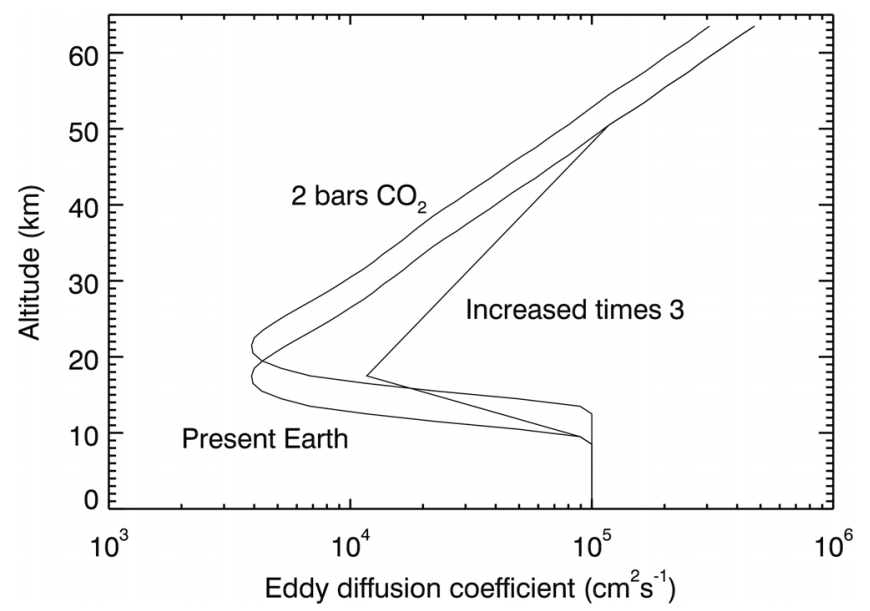

Fig. 2. Eddy diffusion coefficient $\left(k_{\text {edd }}\right)$ profiles used in our simulations. Present Earth $k_{\text {edd }}$ is the one measured by Massie \& Hunten (1981), the other two profiles are modified versions of this coefficient for the 2 bar of $\mathrm{CO}_{2}$ atmosphere and more pausible $k_{\text {edd }}$ for early Earth (see Results).

simulations for higher UV fluxes. As discussed further below, stellar UV fluxes at wavelengths less than $\sim 200 \mathrm{~nm}$ originate from a star's chromosphere and are, if anything, anti-correlated with the flux at longer wavelengths. Stars tend to brighten in the visible as they age; however, their short-wavelength UV emissions decrease with age in most stars because their spin rate slows and they exhibit less chromospheric activity. Longer wavelengths are included in the model, but they mostly affect the temperature profile, which has been fixed for the planets simulated here.

We also did calculations for early Earth-type planets with $\mathrm{CO}_{2}$ partial pressures of $0.02,0.2$, and 2 bars and higher UV radiation than the present solar flux. For the 0.02-bar case, we kept the surface temperature and pressure the same as for the standard model. (This implies slightly more $\mathrm{N}_{2}$ and either a higher incident solar flux or additional unspecified greenhouse warming.) For the 2-bar $\mathrm{CO}_{2}$ case, the total surface pressure was 2.9 bars $\left(=\mathrm{pCO}_{2}+\mathrm{pN}_{2}+\mathrm{pH}_{2} \mathrm{O}\right)$. The surface temperature, $317 \mathrm{~K}$, and the vertical temperature profile for this atmosphere were calculated by a climate model (Kasting 1990). In this latter case, the eddy diffusion profile was readjusted to account for the increased troposphere thickness. Following Kasting (1990) we shifted the eddy diffusion profile upward by $4 \mathrm{~km}$ for the 2 bar case, and assigned an eddy diffusion coefficient of $10^{5} \mathrm{~cm}^{2} \mathrm{~s}^{-1}$ to the 4 lowermost grid points (Fig. 2). Although the cases that we studied are not entirely self-consistent, we chose them because they span a plausible range of atmospheric composition for abiotic, early Earth-type planets (Kasting 1990). Volcanic outgassing of $\mathrm{H}_{2}$ and other reduced species was included in most, but not all, of the model calculations. For $\mathrm{H}_{2}$, we used the present volcanic outgassing rate $\sim 5 \times 10^{12} \mathrm{~mol} \mathrm{yr}^{-1}$, or $\sim 2 \times 10^{10} \mathrm{~cm}^{-2} \mathrm{~s}^{-1}$ (Holland 2002). Sleep \& Bird (2007) argue for an $\mathrm{H}_{2}$ outgassing rate that is lower by a factor of 10 . Our calculations span this range because we consider zero outgassing as well. The methane flux for the standard case was set to the estimated modern non-biogenic flux on hydrothermal vents. Formerly, the biotic-to-abiotic $\mathrm{CH}_{4}$ ratio was estimated to be $\sim 300: 1$ (Kasting \& Catling 2003). New measurements of methane dissolved in the fluids of the Lost City vent field (Kelley et al. 2005) indicate that the abiotic methane flux is higher than previously thought by a factor of 10; therefore, the biotic-to-abiotic ratio may be $\sim 30: 1$. The present biological methane flux is $535 \mathrm{Tg} \mathrm{CH} /$ year or $3.3 \times 10^{13} \mathrm{~mol} / \mathrm{yr}$ 
(Houghton et al. 1995), so the assumed abiotic methane flux was $1 \times 10^{12} \mathrm{~mol} / \mathrm{yr}$, or $3.7 \times 10^{9} \mathrm{~cm}^{-2} \mathrm{~s}^{-1}$. The actual abiotic methane flux may be lower than this value if the $\mathrm{CH}_{4}$ in the vent fluids is produced by methanogens living deep within the vent systems. An (implicit) $\mathrm{NH}_{3}$ flux of $3.87 \times 10^{9} \mathrm{~cm}^{-2} \mathrm{~s}^{-1}$ was included because the standard model contained $10 \mathrm{ppbv}$ of $\mathrm{NH}_{3}$. This was simply for convenience, because $\mathrm{NH}_{3}$ was included in the species list and, hence, its concentration must be non-zero. For the "no-outgassing" case, the methane and $\mathrm{H}_{2}$ fluxes were set equal to zero, but volcanic outgassing of $\mathrm{SO}_{2}$ and a tiny amount of $\mathrm{NH}_{3}$ was retained in order to avoid numerical problems associated with disappearing species within the code. The implications of this assumption are discussed below.

Our model also includes the volcanic outgassing of $\mathrm{SO}_{2}$ at its present estimated release rate $3.5 \times 10^{9} \mathrm{~cm}^{-1} \mathrm{~s}^{-1}$ (Kasting 1990) along with formation of $\mathrm{NO}, \mathrm{O}_{2}$ and $\mathrm{CO}$ by lightning in the troposphere, as described by Kasting (1990). Rainout rates for soluble species are calculated using the method of Giorgi \& Chameides (1985). These rainout terms are added to the normal chemical loss rate for each species. Sulfur gases are removed from the model atmosphere by rainout and surface deposition and by conversion into particulate sulfur and elemental sulfur, followed by rainout and surface deposition of the particles. Rainout lifetimes of high soluble gases (e.g. $\mathrm{H}_{2} \mathrm{SO}_{4}$ ) and of particles was approximately five days. Less soluble gases $\left(\mathrm{SO}_{2}, \mathrm{H}_{2} \mathrm{~S}\right)$ have longer lifetimes against rainout, according to this model. The lifetimes assumed here are appropiate for the modern atmosphere. we assume that the hydrological cycle and, hence, rainout lifetime was similar on early Earth. This assumption seems reasonable, since evaporation (and, thus, precipitation) rates are ultimately controlled by solar heating rates, not by temperature (Holland 1978). Furthermore, the rainout rate of highly soluble gases is determinated by how often it rains, no how much it rains. Soluble species are also removed by direct deposition at the lower boundary, simulating uptake by the ocean. The downward flux of a chemical species $i$ is equal to its number density, $n_{i}$, multiplied by a deposition velocity, $v_{\text {dep }}$. Deposition velocities for different gases are taken from Slinn et al. (1978) and Lee \& Schwartz (1981). Values range from $\sim 0.02 \mathrm{~cm} / \mathrm{s}$ for less reactive species (e.g., $\mathrm{H}_{2} \mathrm{O}_{2}$ ) to $1 \mathrm{~cm} / \mathrm{s}$ for the most reactive species (e.g., OH). The upper limit on $v_{\text {dep }}$ is set by diffusion through the turbulent atmospheric boundary layer, assuming that molecules are absorbed by the surface each time they collide with it.

One may ask how our photochemical model achieves steady state when species are continually injected into the atmosphere from volcanoes. For hydrogen and sulfur, the answer is clear: they are removed either by escape to space (for $\mathrm{H}$ ) or by rainout from the atmosphere. For $\mathrm{CH}_{4}$ and $\mathrm{NH}_{3}$, the answer is less obvious. These species are oxidized photochemically to $\mathrm{CO}_{2}$ and $\mathrm{N}_{2}$, respectively. As the $\mathrm{CO}_{2}$ and $\mathrm{N}_{2}$ concentrations are held constant in the model atmosphere, these species are implicitly lost by reactions of $\mathrm{CO}_{2}$ with surface minerals (to form carbonates) and by unspecified mechanisms of $\mathrm{N}_{2}$ loss (most plausibly biological nitrogen fixation).

\subsection{The atmospheric hydrogen budget}

A key feature of our model that makes it useful for this study is its ability to keep track of the atmospheric hydrogen budget, or redox budget. The basic principle behind this budgeting scheme is simple: when one species is oxidized, another species must be reduced, and vice versa. Alternatively, one could rephrase this principle as requiring that the electron budget of the model atmosphere be balanced.

In practice, it is easiest to keep track of redox balance in terms of the abundance of molecular hydrogen, $\mathrm{H}_{2}$. Following Kasting \& Brown (1998) we define "redox-neutral" species: $\mathrm{H}_{2} \mathrm{O}$ (for $\mathrm{H}$ ), $\mathrm{N}_{2}$ (for $\mathrm{N}$ ), $\mathrm{CO}_{2}$ (for $\mathrm{C}$ ), and $\mathrm{SO}_{2}$ (for $\mathrm{S}$ ). All other species are assigned redox coefficients relative to these gases by determining how much $\mathrm{H}_{2}$ is produced or consumed during their formation from redox neutral species. For example, formation of hydrogen peroxide can be expressed as:

$2 \mathrm{H}_{2} \mathrm{O} \longrightarrow \mathrm{H}_{2} \mathrm{O}_{2}+\mathrm{H}_{2}$.

The coefficient of $\mathrm{H}_{2} \mathrm{O}_{2}$ in the hydrogen budget is therefore -1 , meaning that when one $\mathrm{H}_{2} \mathrm{O}_{2}$ molecule is rained out, one $\mathrm{H}_{2}$ molecule is produced. Similarly, formation of elemental sulfur, $\mathrm{S}_{8}$, can be written as:

$8 \mathrm{SO}_{2}+16 \mathrm{H}_{2} \longrightarrow \mathrm{S}_{8}+16 \mathrm{H}_{2} \mathrm{O}$.

Hence, rainout of $\mathrm{S}_{8}$ particles consumes $16 \mathrm{H}_{2}$ molecules (per $\mathrm{S}_{8}$ molecule), so the redox coefficient of $\mathrm{S}_{8}$ is +16 . Volcanic outgassing of species other than $\mathrm{H}_{2}$ itself is treated in the same manner, i.e., it adds to the total hydrogen outgassing rate according to the appropriate stoichiometry. As mentioned earlier, the standard model includes modest outgassing of $\mathrm{CH}_{4}$ and $\mathrm{NH}_{3}$, in addition to $\mathrm{H}_{2}$. Thus, the total hydrogen outgassing rate can be written as:

$\Phi_{\text {volc }}\left(\mathrm{H}_{2}\right)=\Phi\left(\mathrm{H}_{2}\right)+1.5 \Phi\left(\mathrm{NH}_{3}\right)+4 \Phi\left(\mathrm{CH}_{4}\right)$

where the terms on the right represent the fluxes of the individual reduced species multiplied by their stoichiometric coefficients in the redox budget.

With these definitions in place, the hydrogen budget can be written as:

$\Phi_{\text {volc }}\left(\mathrm{H}_{2}\right)+\Phi_{\text {rain }}(\mathrm{Ox})=\Phi_{\mathrm{esc}}\left(\mathrm{H}_{2}\right)+\Phi_{\text {rain }}(\mathrm{Red})$.

Here, the terms $\Phi_{\text {rain }}(\mathrm{Ox})$ and $\Phi_{\text {rain }}(\mathrm{Red})$ represent the net flux of photochemically produced oxidants and reductants, respectively, from the atmosphere to the ocean. This flux Fincludes both rainout and surface deposition. Equation (1) is diagnostic, not prognostic; hence, it provides a good check both on redox balance and on the photochemical scheme. (This equation will not balance if any of the chemical reactions are not balanced.) In our model, Eq. (1) balances to within one part in $10^{3}$ for the standard case and one part in $10^{5}$ for the other simulated planets (Table 1).

As discussed earlier, we assume that the hydrogen escape is limited by the $\mathrm{H}_{2}$ diffusion rate through the homopause. The diffusion-limited escape flux is given by Hunten (1973) and Walker (1977):

$$
\begin{aligned}
\Phi_{\mathrm{esc}}\left(\mathrm{H}_{2}\right) & =\left(\frac{b}{H}\right) f_{\text {tot }} \\
& \cong 2.5 \times 10^{13} f_{\text {tot }}\left(\text { molecules } \mathrm{cm}^{-2} \mathrm{~s}^{-1}\right)
\end{aligned}
$$

where $b$ is an average binary diffusion coefficient for the diffusion of $\mathrm{H}$ and $\mathrm{H}_{2}$ in nitrogen, $H$ is the atmospheric scale height $(R T / g)$, and $f_{\text {tot }}$ is the sum of the mixing ratios of all the hydrogen-containing species, weighted by the number of hydrogen atoms they contain. As we have expressed the hydrogen budget in terms of $\mathrm{H}_{2}$ molecules, we do the same for $f_{\text {tot }}$ :

$f_{\text {tot }}=f\left(\mathrm{H}_{2}\right)+0.5 f(\mathrm{H})+f\left(\mathrm{H}_{2} \mathrm{O}\right)+\ldots$

Because the stratosphere is cold and dry in all of our simulations, the contribution of $\mathrm{H}_{2} \mathrm{O}$ to $\mathrm{f}_{\text {tot }}\left(\mathrm{H}_{2}\right)$ is negligible. When this is not the case (as in the runaway greenhouse example mentioned earlier), high abiotic $\mathrm{O}_{2}$ concentrations are possible. The actual hydrogen escape rate may be lower than predicted by Eq. (2) However, that is not a concern for this paper, as slower $\mathrm{H}$ escape 
will lead to higher atmospheric $\mathrm{H}_{2}$ concentrations, and hence lower abiotic $\mathrm{O}_{2}$ and $\mathrm{O}_{3}$. When trying to identify "false positives" for life, the conservative approach is to assume the maximum, diffusion-limited, hydrogen escape rate.

\subsection{The input stellar spectra}

Some of the stars observed by the TPF missions and by Darwin may be young stars with UV fluxes higher than that of the Sun. High stellar UV should increase the rate of $\mathrm{CO}_{2}$ photolysis, and thus enhance high-altitude concentrations of $\mathrm{O}_{2}$, and possibly $\mathrm{O}_{3}$, on planets orbiting such stars. High atmospheric $\mathrm{CO}_{2}$ concentrations on young, early Earth-type planets would presumably enhance production of $\mathrm{O}_{2}$ and $\mathrm{O}_{3}$. To determine whether this process might produce enough abiotic $\mathrm{O}_{2}$ and $\mathrm{O}_{3}$ to be detected remotely, we simulated planets with high- $\mathrm{CO}_{2}$ atmospheres around a star with a much higher UV flux than our Sun. EK Draconis was chosen from a suite of solar-type stars studied by Ribas et al. (2005) because it has the highest UV flux of the six stars studied. EK Dra itself may be an unlikely candidate for study by TPF or Darwin because of its extremely young age (see Discussion). However, our goal here was to study a planet exposed to a maximal UV radiation flux to determine if this environment favors abiotic formation of $\mathrm{O}_{2}$ and $\mathrm{O}_{3}$. EK Dra (HD 129333) is a G1.5 V spectral type member of the Pleiades moving group. It has an estimated age of $\sim 0.1 \mathrm{Gyr}$, making it a good proxy for the young Sun (Dorren \& Guinan 1994; Strassmeier \& Rice 1998; Montes et al. 2001; Ribas et al. 2005). Dorren \& Guinan (1994) state that it is the "only known star closely matching the zero age main sequence Sun observable with IUE" (the International Ultraviolet Explorer.)

An absolute stellar spectral energy distribution was assembled from a Kurucz (1993) photospheric spectrum with $T_{\text {eff }}=$ $5765 \mathrm{~K}$, surface gravity $\log g=4.61 \mathrm{~g} \mathrm{~cm}^{-2}$, and solar metallicity. (Gray et al. (2003) derived $[\mathrm{M} / \mathrm{H}]-0.04 \pm 0.09 \mathrm{dex}$ ). The spectrum was absolutely normalized at 21 observed photometric points between 0.38 and $12 \mu \mathrm{m}$, as described by Cohen et al. (2003). Nineteen empirical UV spectra (17 SW, 2 LW) were recalibrated (Massa \& Fitzpatrick 2000) and combined using the overlap. From 0.335 to $160 \mu \mathrm{m}$ the spectrum is the normalized photosphere. Below $0.335 \mu \mathrm{m}$ it is an 11-point boxcar-smoothed version of the empirical UV data.

The Ly $\alpha$ flux has not been measured for EK Dra. Therefore, we estimated its flux using the empirical expression derived by Ribas et al. (2005, Eq. (2)). At the top of the planet's atmosphere, the Ly $\alpha$ flux was estimated to be $105.8 \mathrm{erg} \mathrm{cm}^{-2} \mathrm{~s}^{-1}$. By comparison, the $\operatorname{Ly} \alpha$ flux from our Sun varies from $\sim 6$ to $10 \mathrm{erg} \mathrm{cm}^{-2} \mathrm{~s}^{-1}$ during one activity cycle at $1 \mathrm{AU}$ (Emerich et al. 2005). The simulated planets around EK Dra were positioned so as to receive the same integrated solar flux as present Earth $\left(1375 \mathrm{~W} \mathrm{~m}^{-2}\right)$. This condition is satisfied if the planet's orbital radius is $0.97 \mathrm{AU}$. (EK Dra is slightly less luminous than the present Sun.) The UV flux at the top of the simulated planet's atmosphere is shown in Fig. 3. Earth's UV flux is also shown for comparison. The solar flux used in the code comes from the World Meteorological Organization (1985).

\subsection{Radiative transfer model}

To generate the spectra shown in this paper, the pressure and temperature profiles and calculated mixing ratios from the coupled climate-chemical model are used as input to a line-byline radiative transfer model, which generates angle-dependent

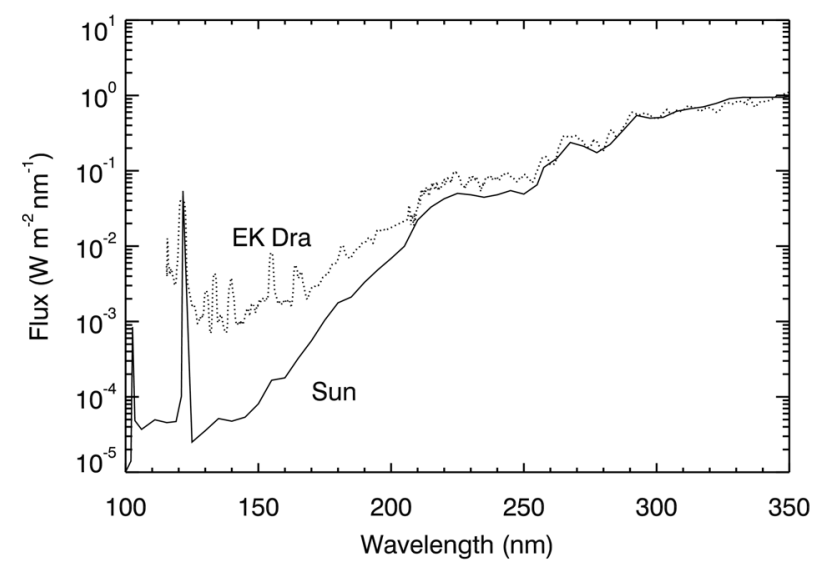

Fig. 3. UV fluxes for the Sun and EK Dra at the top of the atmosphere of a habitable planet at $1 \mathrm{AU}$ equivalent distance.

synthetic radiance spectra. As the first step in this process the temperature and pressure profiles, and the calculated mixing ratios for $\mathrm{H}_{2} \mathrm{O}, \mathrm{O}_{2}, \mathrm{O}_{3}, \mathrm{CO}_{2}$, and $\mathrm{CH}_{4}$ are input to a model (LBLABC: Meadows \& Crisp 1996) which generates line-byline monochromatic gas absorption coefficients. LBLABC employs several nested spectral grids that completely resolve the narrow cores of individual gas absorption lines at all atmospheric levels, and include their contributions at very large distances from the line center (e.g. 500 to $1000 \mathrm{~cm}^{-1}$ from the line centers).

This model was originally developed for simulating radiative processes in the deep atmosphere of Venus (Meadows \& Crisp 1996) and for highly precise simulation of radiances and heating rates in the Earth's atmosphere (Crisp 1997). In addition, since we are working with relatively massive $\mathrm{CO}_{2}$ atmospheres, we explicitly included the effects of collisionallyinduced absorption for $\mathrm{CO}_{2}$. Our current models include $\mathrm{CO}_{2}$ continuum absorption at all wavelengths where it has been measured or predicted. At wavelengths between 33 and $250 \mu \mathrm{m}$, we use the pressure induced rotational absorption (PIA) formulation described by Gruszka \& Borysow (1996-1998). At wavelengths between 4 and $10 \mu \mathrm{m}$, we simulate the $\mathrm{CO}_{2}$ PIA using the formulation described by Kasting et al. (1984b). At near-IR wavelengths, we used the empirical pressure-induced absorption coefficients from Moore (1971).

LBLABC simulates the line shape function differently in the line-center and far-wing regions. For line-center distances less than 40 Doppler halfwidths, a Rautian line shape is used. This line shape incorporates Doppler broadening, collisional (Lorentzian) broadening, and collisional (Dicke) narrowing (cf. Goody \& Yung 1989). At greater distances, a Van VleckWeisskopf profile is used for all gases except for $\mathrm{H}_{2} \mathrm{O}, \mathrm{CO}_{2}$, and $\mathrm{CO}$. The super-Lorentzian behavior of the far wings of $\mathrm{H}_{2} \mathrm{O}$ lines, which has been attributed to the finite duration of collisions, is parameterized by multiplying the Van Vleck-Weisskopf profile by a wavenumber-dependent $\mathrm{X}$ factor (Clough et al. 1989). This profile is adequate to account for the water vapor continuum absorption seen at thermal infrared wavelengths, as well as the weak continuum absorption seen throughout much of the visible and near-infrared spectrum (Crisp 1997). The subLorentzian behavior of the $\mathrm{CO}_{2}$ line profile is primarily a consequence of a vibration-rotation energy redistribution process called collisional line mixing. Direct ab initio methods now exist for computing these effects (cf. Hartmann \& Boulet 1991), but these methods do not yet provide the accuracy or numerical efficiency required for routine use. LBLABC currently employs 
a simple, semi-empirical algorithm to correct for the effects of line mixing. For strongly mixed Q-branch lines, we use the first order correction described by Rosenkranz (1988). With the line mixing correction and the use of a sub-Lorentzian Chi-factor (cf. Fig. 2 of Meadows \& Crisp 1996), these methods provide adequate accuracy for modeling the $\mathrm{CO}_{2}$ absorption at pressures and temperatures as high as those seen in the deep atmosphere of Venus (90-bars), and should provide the accuracy needed for the calculations proposed here.

These products, along with the atmospheric properties and constituent mixing ratios were used as input into our line-byline radiative transfer model, SMART_SPECTRA, which was developed by the Virtual Planetary Laboratory for use with generalized terrestrial planet climate models, and is an updated version of the Spectral Mapping Atmospheric Radiative Transfer model (SMART; Meadows \& Crisp 1996; Crisp 1997) developed by D. Crisp. For this application, SMART_SPECTRA was used to calculate a high resolution spectrum of each planet for a solar zenith angle of $60^{\circ}$, which approximates the average illumination observed in a planetary disk-average. All spectra were generated over an ocean surface, which provides a neutral background for understanding the atmospheric changes in the models. The ocean surface, however, is extremely dark; thus, the reflectivities shown here will typically be much lower than would be expected for the present Earth disk-average, which would also include the higher-reflectivity contributions from continents and clouds. For the visible and near-infrared spectra shown here, the albedo is computed as the ratio of the integrated upward flux over the hemisphere, divided by $\pi$ steradians, to approximate the mean radiance seen in the disk-average, and then divided by the solar insolation at the top of the atmosphere. For the mid-infrared spectra, the radiance shown is the integrated upward flux over the hemisphere, divided by $\pi$ steradians.

\section{Results}

\subsection{Early Earth standard atmosphere}

The vertical profiles of $\mathrm{O}_{2}$ and $\mathrm{O}_{3}$ obtained in our "standard" atmosphere ( 0.2 bars $\mathrm{CO}_{2}$ planet around the Sun) are presented in Fig. 4, and the associated columns depths are summarized in Table 2.

To illustrate the role of volcanic outgassing of reduced gases in determining $\mathrm{O}_{2}$ and $\mathrm{O}_{3}$ concentrations, we performed a simulation with $\mathrm{H}_{2}$ and $\mathrm{CH}_{4}$ outgassing turned off. As expected, more $\mathrm{O}_{2}$ and $\mathrm{O}_{3}$ were formed in the no-outgassing case. The calculated increases relative to the standard case were: $\sim 4$ times for $\mathrm{O}_{2}$ and a factor of $\sim 40$ for $\mathrm{O}_{3}$ (Table 2). The latter increase is, of course, large in a relative sense; however, the absolute ozone column density for the no-outgassing case is still $<0.1 \%$ of the mean ozone column density on present Earth. The reason why the calculated increases in $\mathrm{O}_{2}$ and $\mathrm{O}_{3}$ were so modest is that $\mathrm{H}_{2}$ did not disappear from the atmosphere even though volcanic outgassing was turned off (Fig. 4a). The $\mathrm{H}_{2}$ remaining in this no-outgassing case is produced by atmospheric photochemistry, followed by rainout of oxidized species from the model atmosphere (Table 1).

A set of sensitivity tests was performed in order to quantify some of the uncertainties in our model. Most of these test were done for the "no outgassing" case, which maximizes the amount of abiotic $\mathrm{O}_{2}$ and $\mathrm{O}_{3}$. Five specific modifications were tested in the model, the results of which are listed in Table 2. First, we doubled the third body reaction rates for $\mathrm{O}_{3}$ and $\mathrm{O}_{2}$ formation to simulate the possible effects of having $\mathrm{CO}_{2}$ as a third body. This
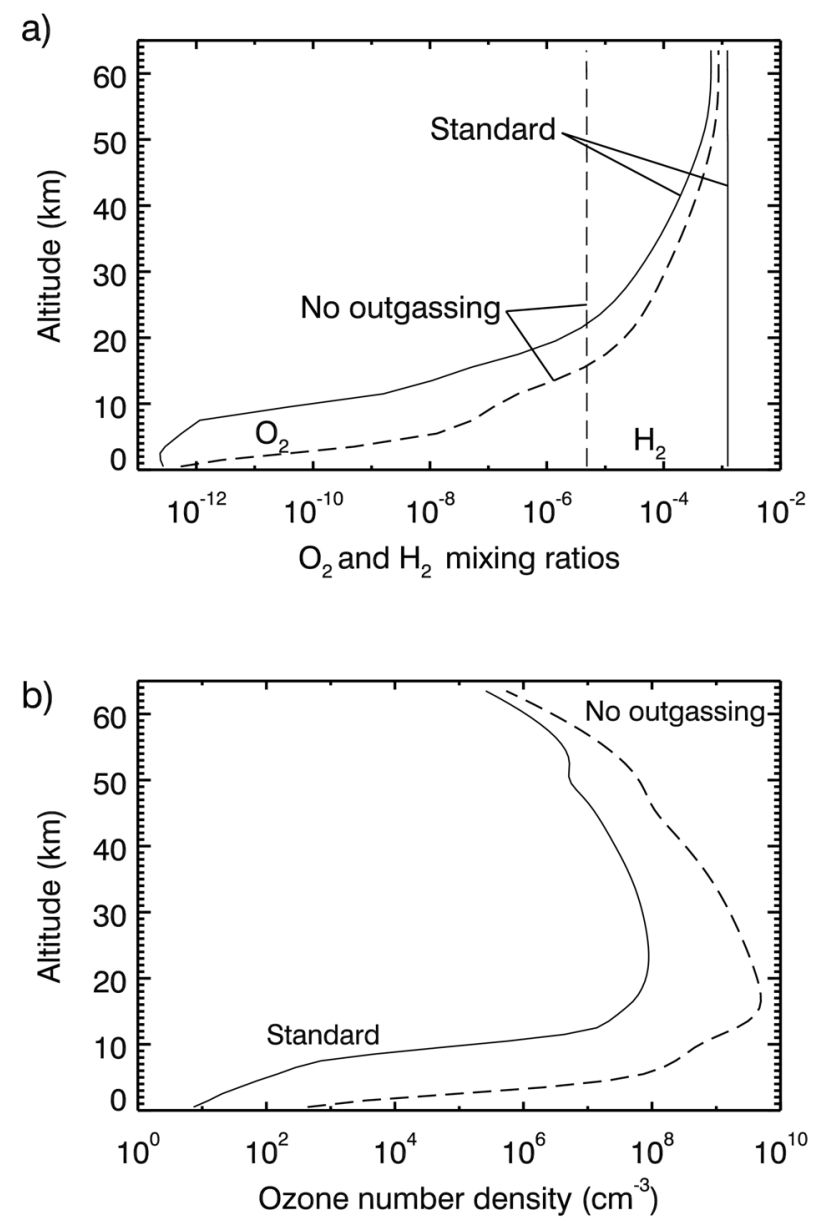

Fig. 4. $\mathrm{O}_{2}$ and $\mathrm{H}_{2}$ mixing ratios a) and $\mathrm{O}_{3}$ number densities b) for the standard and no outgassing cases.

caused a modest $(\sim 30 \%)$ increase in the column depth of $\mathrm{O}_{3}$ and actually decreased the column depth of $\mathrm{O}_{2}$. Second, we eliminated the water absorption for wavelengths greater than $195 \mathrm{~nm}$ where the absorption coefficients have been extrapolated from measurements at shorter wavelengths. This caused a 2-fold increase in both $\mathrm{O}_{2}$ and $\mathrm{O}_{3}$, presumably due to the slower recombination of $\mathrm{O}_{2}$ and $\mathrm{H}_{2}$ when the abundance of $\mathrm{H}$ and $\mathrm{OH}$ radicals (from $\mathrm{H}_{2} \mathrm{O}$ photolysis) is reduced. Third, the minimum of the eddy diffusion coefficient $\left(k_{\text {edd }}\right)$ was increased by 3 (Fig. 2$)$ in order to simulate the effect of no having an inversion layer due to the lack of $\mathrm{O}_{3}$ heating in the atmosphere as explained by Kasting et al. (1979). This decreased $\mathrm{O}_{2}$ by about $40 \%$ as a consequence of faster transport of $\mathrm{O}_{2}$ from the upper stratosphere, where it is produced from $\mathrm{CO}_{2}$ photolysis, to the troposphere, where it is consumed by reaction with $\mathrm{H}_{2}$. We then raised and lowered the assumed stratospheric temperature by 20 degrees. Lowering the stratospheric temperature raised the $\mathrm{O}_{3}$ column depth by $\sim 30 \%$, while raising it lowered the column depth by a factor of 2 . Both effects are caused by the increased rate of $\mathrm{O}_{3}$ destruction at high temperatures.

We also performed one additional sensitivity test in which the planet's surface temperature was reduced to $240 \mathrm{~K}$. This is similar to the "Snowball Earth" simulation of Liang et al. (2006). In this case, we did not turn off volcanic outgassing of reduced gases, because as noted in the Introduction, this is one of the known "false positive" that could lead to arbitrarily high levels of atmospheric $\mathrm{O}_{2}$. Liang et al. did not predict high $\mathrm{O}_{2}$ levels, but that is because they assumed that photochemically 
Table 1. Hydrogen budget for planets around the Sun and EK Dra (high UV) with 0.2 bars of $\mathrm{CO}_{2}$.

\begin{tabular}{|c|c|c|c|c|}
\hline \multirow{2}{*}{$\begin{array}{l}\text { Chemical } \\
\text { species }\end{array}$} & \multirow{2}{*}{$\begin{array}{l}\text { Redox } \\
\text { coefficient }\end{array}$} & \multicolumn{3}{|c|}{$\mathrm{H}_{2}$ budget contribution ${ }^{a}$} \\
\hline & & Standard & No outgassing & High UV \\
\hline \multicolumn{5}{|c|}{ Reduced species } \\
\hline $\mathrm{H}$ & 0.5 & $4.88 \times 10^{5}$ & $8.80 \times 10^{6}$ & $2.47 \times 10^{5}$ \\
\hline $\mathrm{CO}$ & 1 & $3.58 \times 10^{7}$ & $3.02 \times 10^{5}$ & $2.04 \times 10^{7}$ \\
\hline $\mathrm{HCO}$ & 1.5 & $1.42 \times 10^{8}$ & $4.45 \times 10^{7}$ & $2.18 \times 10^{8}$ \\
\hline $\mathrm{H}_{2} \mathrm{CO}$ & 2 & $3.68 \times 10^{9}$ & $3.34 \times 10^{8}$ & $9.16 \times 10^{9}$ \\
\hline $\mathrm{H}_{2} \mathrm{~S}$ & 3 & $2.16 \times 10^{8}$ & $3.84 \times 10^{5}$ & $1.79 \times 10^{8}$ \\
\hline HS & 2.5 & $8.40 \times 10^{7}$ & $5.97 \times 10^{5}$ & $6.40 \times 10^{7}$ \\
\hline $\mathrm{HSO}$ & 1.5 & $7.36 \times 10^{7}$ & $3.49 \times 10^{5}$ & $4.55 \times 10^{7}$ \\
\hline $\mathrm{NH}_{2}$ & 1 & $8.02 \times 10^{6}$ & $6.75 \times 10^{2}$ & $9.04 \times 10^{6}$ \\
\hline $\mathrm{N}_{2} \mathrm{H}_{3}$ & 1.5 & $1.24 \times 10^{7}$ & $8.10 \times 10^{-1}$ & $1.47 \times 10^{7}$ \\
\hline $\mathrm{N}_{2} \mathrm{H}_{4}$ & 2 & $1.50 \times 10^{8}$ & $5.55 \times 10^{-1}$ & $1.23 \times 10^{8}$ \\
\hline$\Phi_{\text {rain }}($ Red $)$ & & $4.4 \times 10^{9}$ & $3.89 \times 10^{8}$ & $9.83 \times 10^{9}$ \\
\hline \multicolumn{5}{|c|}{ Oxidized species } \\
\hline $\mathrm{O}$ & -1 & $2.00 \times 10^{5}$ & $5.91 \times 10^{6}$ & $6.83 \times 10^{5}$ \\
\hline $\mathrm{HO}_{2}$ & -1.5 & $6.56 \times 10^{6}$ & $6.73 \times 10^{6}$ & $6.97 \times 10^{6}$ \\
\hline $\mathrm{H}_{2} \mathrm{O}_{2}$ & -1 & $7.28 \times 10^{5}$ & $2.68 \times 10^{7}$ & $1.13 \times 10^{6}$ \\
\hline $\mathrm{HNO}$ & -0.5 & $2.63 \times 10^{7}$ & $2.96 \times 10^{8}$ & $3.47 \times 10^{7}$ \\
\hline $\mathrm{H}_{2} \mathrm{SO}_{4}$ & -1 & $8.64 \times 10^{5}$ & $6.97 \times 10^{6}$ & $2.21 \times 10^{6}$ \\
\hline $\mathrm{SO}_{4}$ aerosol & -1 & $8.47 \times 10^{7}$ & $1.88 \times 10^{8}$ & $1.55 \times 10^{8}$ \\
\hline$\Phi_{\text {rain }}(\mathrm{Ox})$ & & $1.19 \times 10^{8}$ & $5.31 \times 10^{8}$ & $2.01 \times 10^{8}$ \\
\hline \multicolumn{5}{|c|}{ Volcanism contribution } \\
\hline $\mathrm{H}_{2}$ & 1 & $2.00 \times 10^{10}$ & 0.0 & $2.00 \times 10^{10}$ \\
\hline $\mathrm{NH}_{3}$ & 1.5 & $3.87 \times 10^{9}$ & $5.27 \times 10^{6}$ & $4.21 \times 10^{9}$ \\
\hline $\mathrm{CH}_{4}$ & 4 & $1.80 \times 10^{10}$ & 0.0 & $1.80 \times 10^{10}$ \\
\hline$\Phi_{\text {volc }}\left(\mathrm{H}_{2}\right)$ & & $4.19 \times 10^{10}$ & $5.27 \times 10^{6}$ & $4.22 \times 10^{10}$ \\
\hline$\Phi_{\mathrm{esc}}\left(\mathrm{H}_{2}\right)$ & & $3.76 \times 10^{10}$ & $1.47 \times 10^{8}$ & $3.26 \times 10^{10}$ \\
\hline $\mathrm{H}_{2}$ balance & & $3.67 \times 10^{7}$ & $6.02 \times 10^{3}$ & $3.53 \times 10^{5}$ \\
\hline
\end{tabular}

${ }^{a}$ In molecules $\mathrm{cm}^{-2} \mathrm{~cm}^{-1}$.

Table 2. $\mathrm{O}_{3}$ and $\mathrm{O}_{2}$ column depths $\left(\mathrm{cm}^{-2}\right)$ and $\mathrm{H}_{2}$ and $\mathrm{CH}_{4}$ volume mixing ratios for high $\mathrm{CO}_{2}$ terrestrial atmospheres. Present Earth values are shown for comparison.

\begin{tabular}{lcccc}
\hline \hline $\begin{array}{l}\text { Case } \\
\left.\text { (bars of } \mathrm{CO}_{2}\right)\end{array}$ & $\begin{array}{c}\text { Ozone } \\
\text { column depth }\end{array}$ & $\begin{array}{c}\text { Oxygen }\left(\mathrm{O}_{2}\right) \\
\text { column depth }\end{array}$ & $\begin{array}{c}\text { Hydrogen } \\
\text { mixing ratio }\end{array}$ & $\begin{array}{c}\text { Methane } \\
\text { mixing ratio }\end{array}$ \\
\hline Present $U V$ & $8.61 \times 10^{18}$ & $4.65 \times 10^{24}$ & $5.5 \times 10^{-7}$ & $1.6 \times 10^{-6}$ \\
Present Earth & $1.87 \times 10^{14}$ & $1.24 \times 10^{19}$ & $1.25 \times 10^{-3}$ & $1.38 \times 10^{-4}$ \\
0.2 standard & $7.51 \times 10^{15}$ & $4.94 \times 10^{19}$ & $4.85 \times 10^{-6}$ & $9.4 \times 10^{-12}$ \\
0.2 no outgassing & & & & \\
Sensitivity tests & & & \\
No outgassing case modified & & & & \\
$\mathrm{O}_{2}$ \& O O $_{3}$ third body reactions & $1.09 \times 10^{16}$ & $4.51 \times 10^{19}$ & $5.2 \times 10^{-6}$ & $9.2 \times 10^{-12}$ \\
$\mathrm{H}_{2} \mathrm{O}$ absorption eliminated & $1.65 \times 10^{16}$ & $8.34 \times 10^{19}$ & $4.0 \times 10^{-8}$ & $2.1 \times 10^{-16}$ \\
$k_{\text {edd } \text { increased }}$ & $6.84 \times 10^{15}$ & $2.78 \times 10^{19}$ & $3.8 \times 10^{-6}$ & $1.0 \times 10^{-11}$ \\
Stratospheric temperature 200 K & $3.66 \times 10^{15}$ & $4.38 \times 10^{19}$ & $5.8 \times 10^{-6}$ & $7.4 \times 10^{-12}$ \\
Stratospheric temperature 160 K & $1.05 \times 10^{16}$ & $4.64 \times 10^{19}$ & $4.7 \times 10^{-6}$ & $8.6 \times 10^{-12}$ \\
Outgassing case modified & & & & \\
Surface temperature = 240 K & $3.45 \times 10^{13}$ & $2.24 \times 10^{18}$ & $9.4 \times 10^{-4}$ & $2.7 \times 10^{-4}$ \\
Higher UV & & & & \\
0.02 & $3.53 \times 10^{14}$ & $6.62 \times 10^{18}$ & $8.29 \times 10^{-4}$ & $1.66 \times 10^{-5}$ \\
0.2 & $6.79 \times 10^{14}$ & $3.63 \times 10^{19}$ & $1.09 \times 10^{-3}$ & $4.07 \times 10^{-5}$ \\
2.0 & $5.13 \times 10^{14}$ & $4.24 \times 10^{19}$ & $1.17 \times 10^{-3}$ & $8.75 \times 10^{-5}$ \\
\hline
\end{tabular}

${ }^{a}$ Value for present Earth from McClatchey et al. (1971). ${ }^{b}$ See text for detailed descriptions.

produced oxidants, specifically $\mathrm{H}_{2} \mathrm{O}_{2}$, would accumulate in the ice. Clearly, that could not continue indefinitely, and so such a simulation does not represent a true steady state. In our simulation, with reduced volcanic gases assumed to be present, the predicted concentrations of $\mathrm{O}_{2}$ and $\mathrm{O}_{3}$ were both low (Table 2). However, abiotic $\mathrm{CH}_{4}$ concentrations increased to $\sim 2.7 \times 10^{-3}$ in this simulation, as a consequence of the reduced concentrations of tropospheric $\mathrm{H}_{2} \mathrm{O}$ and correspondingly low number densities of $\mathrm{OH}$ radicals. Hence, this simulation reinforces the thought that one should be cautious about assuming that $\mathrm{CH}_{4}$ by itself is a bioindicator, as there clearly are situations in which abiotic $\mathrm{CH}_{4}$ might become abundant. 
a)

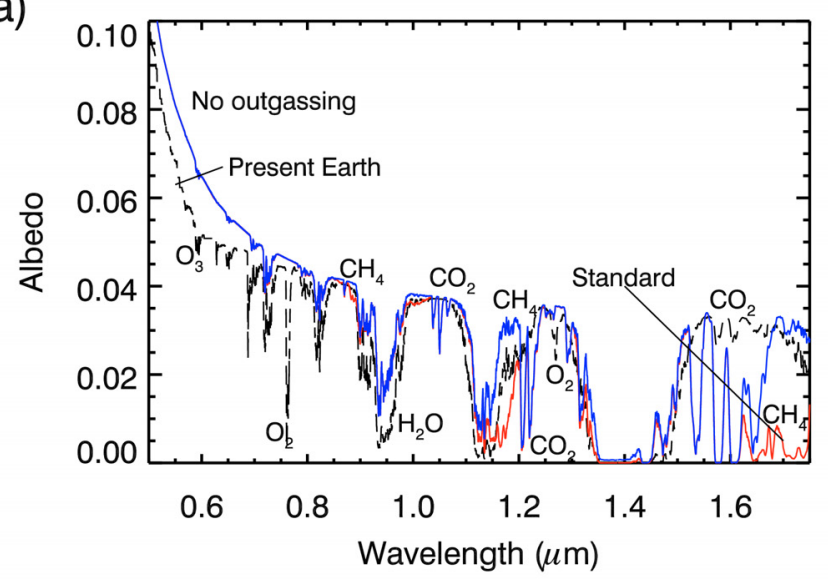

b)

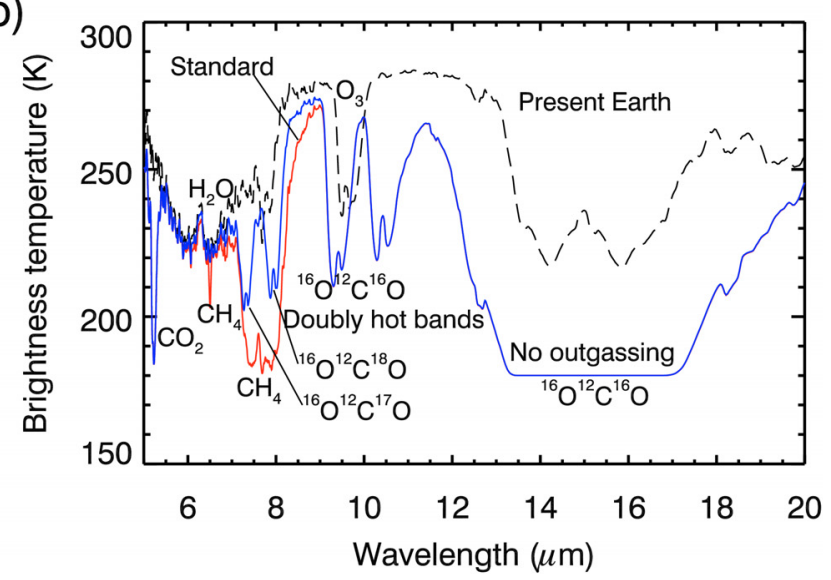

Fig. 5. Spectra of planets with 0.2 bars of $\mathrm{CO}_{2}$ around the Sun. Standard case (red) and no outgassing case (dark blue). Present Earth spectrum is plotted for comparison (dashed line).

Although the sensitivity tests performed here by no means exhaust the possible sources of uncertainty in the photochemical model, they show that most such uncertainties lead to only modest changes in the results. Even a factor of 4-5 uncertainty in the predicted $\mathrm{O}_{2}$ and $\mathrm{O}_{3}$ concentrations is not signficant if the resulting column depths remain well below the level of detection. In general, the results are more sensitive to the boundary conditions imposed on the model, e.g., outgassing and escape of hydrogen, than they are to the details of the model photochemistry.

We also generated spectra at visible and mid-infrared wavelengths for the 0.2 bar $\mathrm{CO}_{2}$ standard early Earth case, both with and without outgassing, and we compared these to the spectrum of modern day Earth (Fig. 5). In the visible-near-IR spectra (Fig. 5a), the principal differences between the two early Earth cases can be seen in the $0.86-0.89 \mu \mathrm{m}, 0.99-1.03 \mu \mathrm{m}, 1.15$ $1.20 \mu \mathrm{m}$ and $1.62-1.70 \mu \mathrm{m}$ wavelength ranges, where the early Earth with outgassing shows more pronounced absorption from methane, as is expected given that the no-outgassing case has a $\sim 10^{7}$ times smaller concentration of this compound (Table 2). For $\mathrm{O}_{2}$, although the no-outgassing case resulted in a 4-fold enhancement in the column depth, the total amount of $\mathrm{O}_{2}$ was still sufficiently small that there is no sign of any absorption from the $\mathrm{O}_{2}$ A-band at $0.76 \mu \mathrm{m}$ in either case. Comparison with the modern Earth spectrum also shows a significant and near-identical reduction in $\mathrm{O}_{3}$ absorption $(0.5-0.7 \mu \mathrm{m})$ for both early Earth cases. Again, the higher $\mathrm{O}_{3}$ column depth for the no-outgassing case was still too small to produce a discernible difference in the spectrum. The early Earth spectra also show strong $\mathrm{CO}_{2}$ absorption in a triplet of bands centered around $1.05 \mu \mathrm{m}$ and a doublet of bands from $1.2-1.25 \mu \mathrm{m}$, features that are absent from the modern Earth spectrum. The strongest $\mathrm{CO}_{2}$ absorption however is seen from $1.52-1.65 \mu \mathrm{m}$, and is present in the modern Earth spectrum, although at much reduced strength.

In the mid-infrared (Fig. 5b), again, the differences in the two early Earth spectra are dominated by methane absorption near $7.7 \mu \mathrm{m}$, which is much more pronounced for the outgassing case. Any difference in the ozone band at $9.6 \mu \mathrm{m}$, however, is masked by the $\mathrm{CO}_{2}$ doubly-hot bands at $9.4 \mu \mathrm{m}$, as noted by Selsis et al. 2002. In comparison to the modern Earth spectrum, the early Earth MIR spectrum is dominated by the absorption of $\mathrm{CO}_{2}$ at 5.3, 7.4, 8.0, 9.4, 10.5 and $15 \mu \mathrm{m}$. These bands are associated with different isotopes of $\mathrm{CO}_{2}$. The $v_{2}$ fundamental of the dominant $\mathrm{CO}_{2}$ isotope $\left({ }^{16} \mathrm{O}^{12} \mathrm{C}^{16} \mathrm{O}\right)$ produces the strongest feature at $15 \mu \mathrm{m}$, which has nearly doubled in apparent width for the 0.2 bar $\mathrm{CO}_{2}$ case, when compared to Modern Earth. The bands at 9.4 and $10.4 \mu \mathrm{m}$ are "doubly hot bands" of the dominant isotope, transitions from upper states to the 2nd excited state, rather than the ground state. However, the bands near 7.4 and $8 \mu \mathrm{m}$ are not hot bands, but rather $v_{1}$ fundamentals for the asymmetric $\mathrm{CO}_{2}$ isotopes $\left({ }^{16} \mathrm{O}^{12} \mathrm{C}^{17} \mathrm{O}\right.$ and $\left.{ }^{16} \mathrm{O}^{12} \mathrm{C}^{18} \mathrm{O}\right)$. This band is forbidden by a Fermi resonance in all symmetric $\mathrm{CO}_{2}$ isotopes, which includes the dominant ${ }^{16} \mathrm{O}^{12} \mathrm{C}^{16} \mathrm{O}$ molecule. The flat base of the $15 \mu \mathrm{m} \mathrm{CO} v_{2} v_{2}$ absorption in the early Earth case is produced by the isothermal stratosphere in our model, which will also serve to enhance the apparent molecular absorption in the spectrum. Should the planet's actual vertical temperature profile deviate from this assumption, then the observed features in the MIR may be less pronounced, even given the same atmospheric composition. Collisionally-induced absorption was also included in the model used to generate these spectra, but it shows relatively little effect on the spectrum throughout this wavelength range at these $\mathrm{CO}_{2}$ partial pressures.

\subsection{High $\mathrm{CO}_{2}$ planetary atmospheres under high UV radiation}

In this experiment, we simulated three planetary atmospheres, each with $0.02,0.2$ and 2 bars of $\mathrm{CO}_{2}$, with high incoming UV radiation. The purpose was to explore the effects of high UV insolation on abiotically produced $\mathrm{O}_{2}$ and $\mathrm{O}_{3}$ in high- $-\mathrm{CO}_{2}$ atmospheres. For the 0.2 bar- $\mathrm{CO}_{2}$ atmosphere under high $\mathrm{UV}$, the column depths of $\mathrm{O}_{2}$ and $\mathrm{O}_{3}$ were $\sim 3$ times that of the comparable standard case with solar insolation (Table 2, Fig. 6). These concentrations, however, are still very low compared with the present Earth's ozone and oxygen column depths. $\mathrm{H}_{2}$ amounts in the standard and high UV atmospheres were very similar (Fig. 6a), but larger photolysis rates of $\mathrm{CO}_{2}$, resulted in more $\mathrm{O}_{2}$ (and hence, $\mathrm{O}_{3}$ ) in the planet's stratosphere (see Fig. 7a).

The ozone column depth changes very little when the $\mathrm{CO}_{2}$ rises from 0.02 bars to 0.2 bars, given the same amount of UV radiation received at the top of the atmosphere (Fig. 8). But, when the $\mathrm{CO}_{2}$ is increased to 2 bars in a high UV environment, the ozone column depth decreases to 0.76 times that in the planet with 0.2 bar $\mathrm{CO}_{2}$ around EK Dra. The likely reason is that $\mathrm{CO}_{2}$ photolysis occurred higher in the stratosphere, where densities are low and where 3-body formation of $\mathrm{O}_{2}$ and $\mathrm{O}_{3}$ is inhibited (Fig. 7). In any case, the calculated changes in $\mathrm{O}_{2}$ and $\mathrm{O}_{3}$ are too small to produce an appreciable effect on the spectra. By contrast, the result of going from the low-UV to the high-UV environment, keeping the same concentration of $\mathrm{CO}_{2}$ in the planetary 
a)
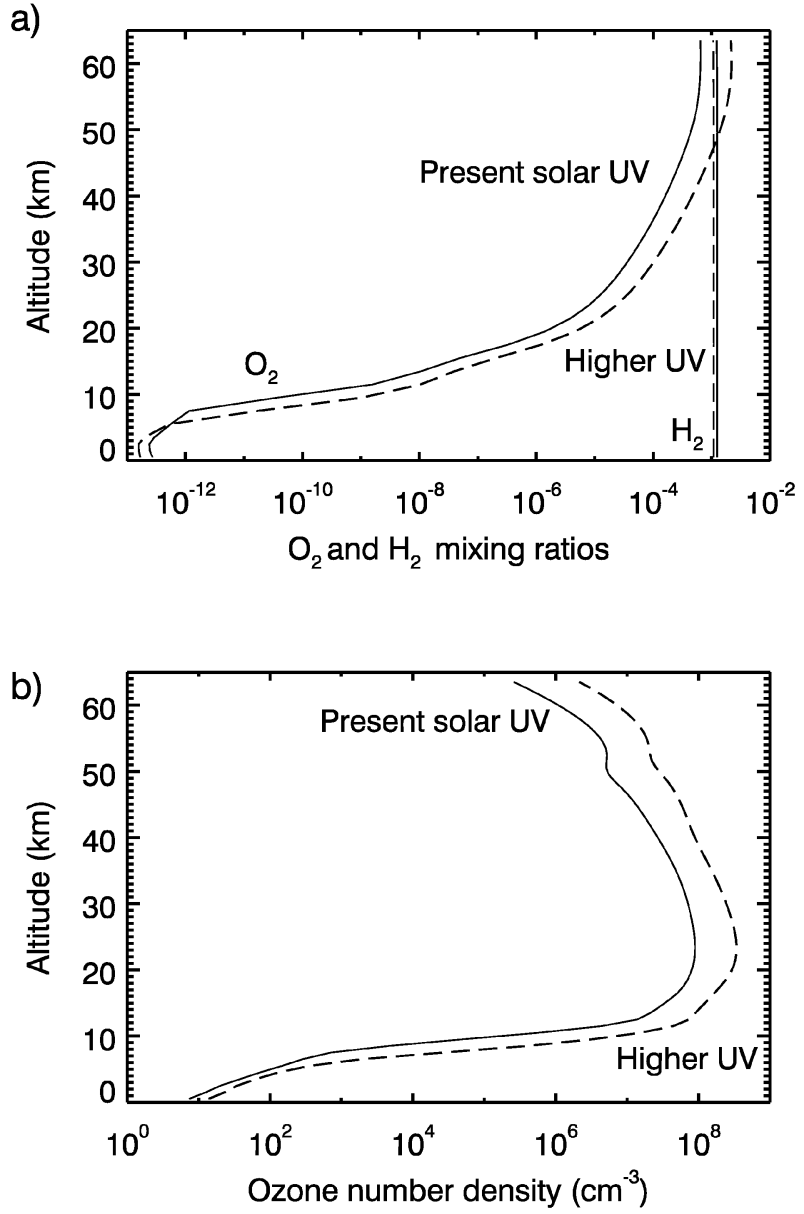

Fig. 6. $\mathrm{O}_{2}$ mixing ratios a) and $\mathrm{O}_{3}$ number densities b) for atmospheres with 0.2 bars of $\mathrm{CO}_{2}$ under present UV solar radiation and high UV flux from a solar-like young star.

atmosphere, is to increase $\mathrm{O}_{2}$ and $\mathrm{O}_{3}$ column depths by a factor of $\sim 3$ (Table 2). Because the planet with the atmosphere containing 2-bars of $\mathrm{CO}_{2}$ had a surface $T(312 \mathrm{~K})$ significantly higher than our 0.02 and 0.2 bar cases $(278 \mathrm{~K})$, we also simulated a planet around EK Dra with 2 bars of $\mathrm{CO}_{2}$, but using the colder surface temperature of $278 \mathrm{~K}$ (Fig. 1). This case was done to minimize the effect of the temperature on the ozone and oxygen destruction and to model an atmosphere more similar to early Mars. The $\mathrm{O}_{3}$ and $\mathrm{O}_{2}$ column depths for this case were less than those obtained for the hotter 2 bar- $\mathrm{CO}_{2}$ planet around EK Dra, being $3.7 \times 10^{14} \mathrm{~cm}^{-2}$ and $2.7 \times 10^{19} \mathrm{~cm}^{-2}$, respectively.

The spectra for these three high-UV cases (Figs. 9 to 11) do show differences from the standard model spectrum. The most obvious difference in the visible spectrum is the change in the slope of the spectrum between $0.5-0.7 \mu \mathrm{m}$. This slope can be affected by both $\mathrm{O}_{3}$ absorption and Rayleigh scattering from atmospheric gases. Since the total $\mathrm{O}_{3}$ column depth for the 0.02 , 0.2 and 2-bar $\mathrm{CO}_{2}$ atmospheres is nearly identical, the difference in the reflectivity in this wavelength range is instead caused by enhanced Rayleigh scattering, and is not the result of differences in ozone absorption. This scattering enhancement is due to either changes in total atmospheric pressure, or atmospheric composition The more massive 2-bar $\mathrm{CO}_{2}$ atmosphere has a surface pressure of 2.9 bars, compared with 1 bar for both the 0.02 and 0.2 bar $\mathrm{CO}_{2}$ cases, and its large enhancement in the Rayleigh scattering slope between $0.5-0.7 \mu \mathrm{m}$ is due primarily to its larger atmospheric pressure. However, the 0.02 and 0.2 bar $\mathrm{CO}_{2}$ a)
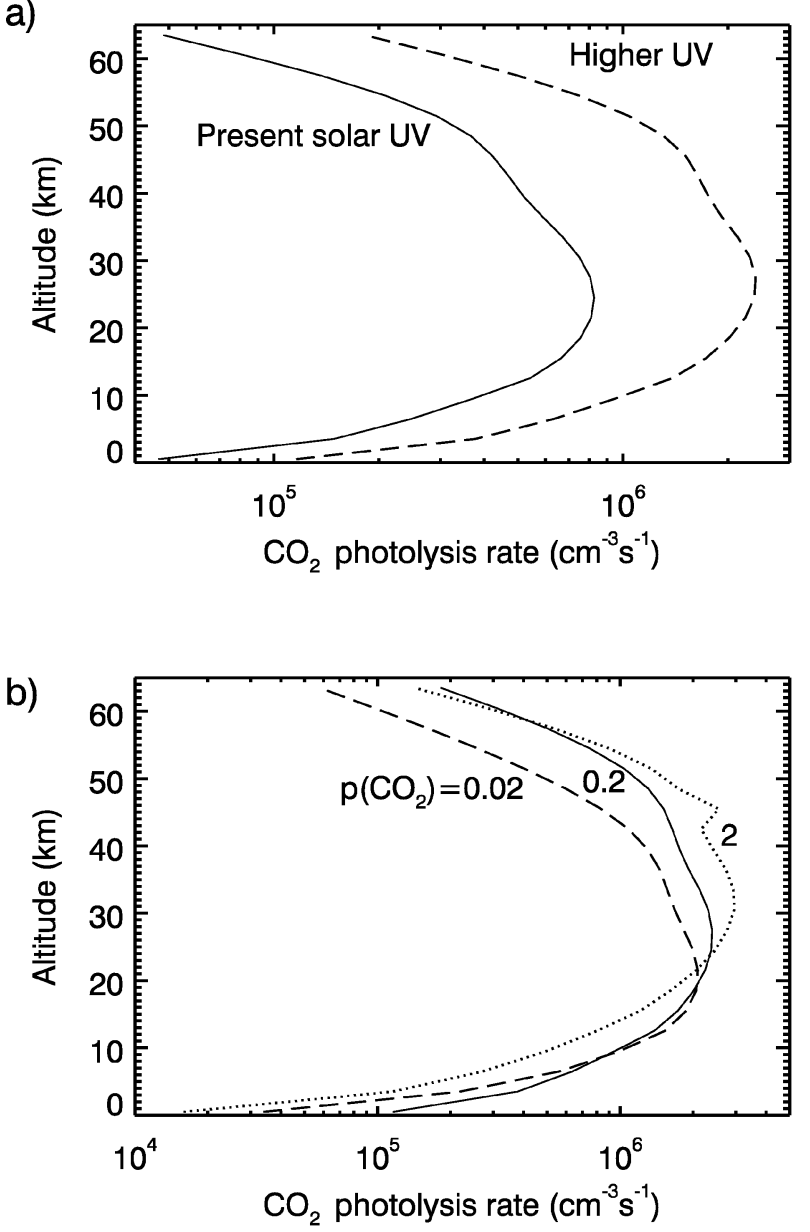

Fig. 7. $\mathrm{CO}_{2}$ photolysis rates for a) atmospheres with 0.2 bar of $\mathrm{CO}_{2}$ under present UV solar radiation and high UV flux from a solar-like young star, and b) atmospheres under high UV radiation with different amounts of $\mathrm{CO}_{2}$.

atmosphere cases also show a small difference in their Rayleigh scattering slopes, even though they have identical atmospheric pressures. This is due instead to the difference in the composition of their atmospheres, as $\mathrm{CO}_{2}$ is a more efficient Rayleigh scatterer than nitrogen, and enhances the Rayleigh scattering slope for the 0.2 bar $\mathrm{CO}_{2}$ case over the 0.02 bar case. In addition to the Rayleigh scattering slope, $\mathrm{CO}_{2}$ absorption features are also seen strongly in the spectrum and reveal spectral sensitivity to atmospheric $\mathrm{CO}_{2}$. The triplet of absorption bands near $1.05 \mu \mathrm{m}$ produces no appreciable absorption for the 0.02 bar case, but can be distiguished as a triplet at high resolution $(R \sim 140$ and above) for both the 0.2 and 2-bar $\mathrm{CO}_{2}$ cases (Fig. 9 - inset). At resolutions of $R \sim 50$ and greater, the triplet structure cannot be clearly discerned, but the ability to distinguish between the 2-bar $\mathrm{CO}_{2}$ and the other two $\mathrm{CO}_{2}$ concentration cases at $1.05 \mu \mathrm{m}$ is maintained (Fig. 10), assuming high $\mathrm{S} / \mathrm{N}$ measurements. The $\mathrm{CO}_{2}$ bands at 1.206 and $1.221 \mu \mathrm{m}$ become progressively stronger as the atmospheric $\mathrm{CO}_{2}$ abundance is increased and can also be distinguished as two features at $R \sim 140$, but still retain sensitivity to atmospheric $\mathrm{CO}_{2}$ abundance at resolutions as low as $R \sim 50$. A similar trend is shown for the stronger $\mathrm{CO}_{2}$ bands near $1.6 \mu \mathrm{m}$, however in this case, the 2-bar $\mathrm{CO}_{2}$ atmosphere is so heavily absorbing that the planetary spectrum becomes black throughout much of the $1.52-1.7 \mu \mathrm{m}$ region, with the exception of a relatively transparent, narrow atmospheric window at $1.55 \mu \mathrm{m}$. 
a)
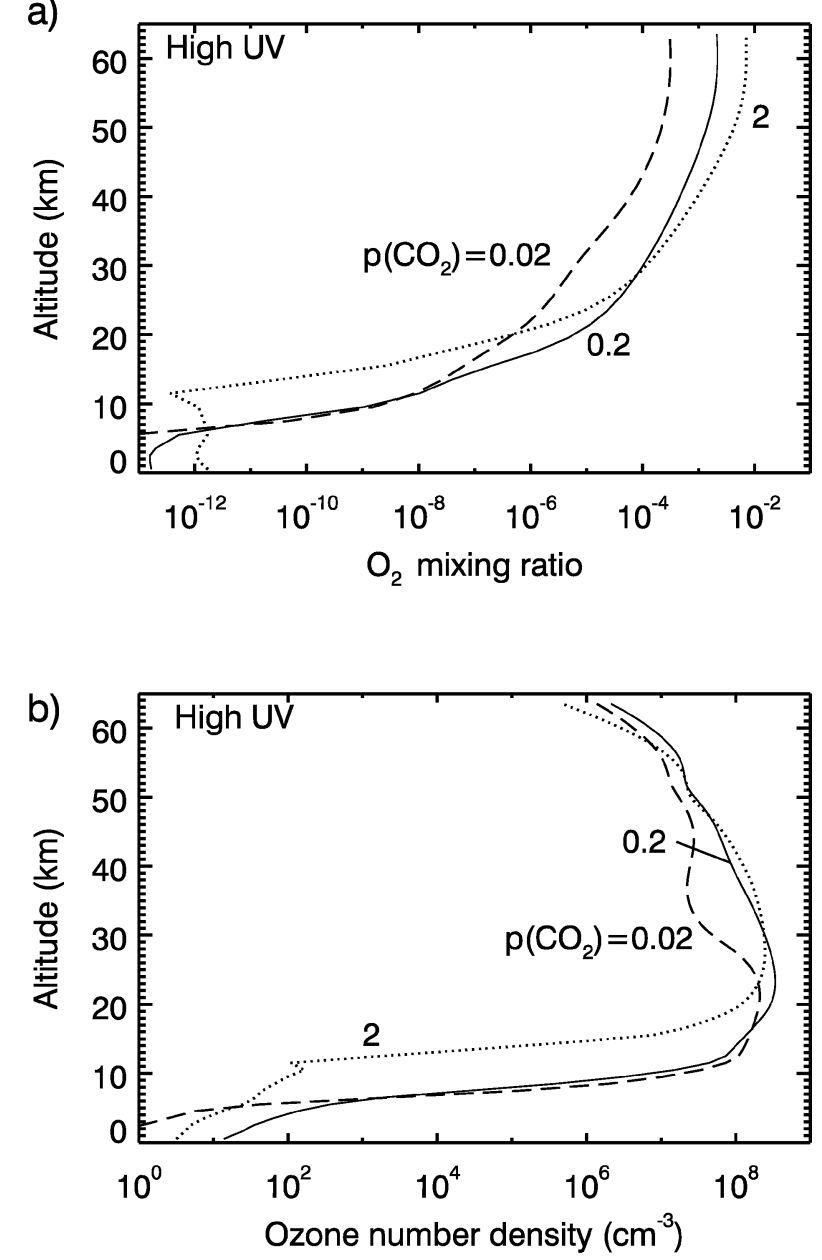

Fig. 8. $\mathrm{O}_{2}$ mixing ratios a) and $\mathrm{O}_{3}$ number densities b) for atmospheres with different amounts of $\mathrm{CO}_{2}$ under high UV flux from a solar-like young star.

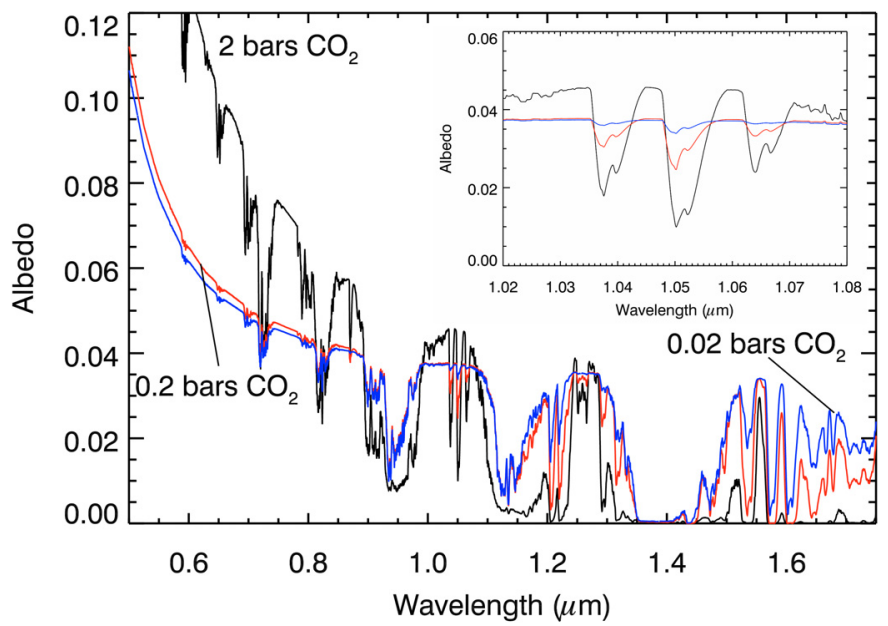

Fig. 9. Visible-NIR spectra of planets with 0.02 (dark blue), 0.2 (red) and 2 bars (black) of $\mathrm{CO}_{2}$ around EK Dra.

Finally, marked differences in the strengths of the water bands near $0.94 \mu \mathrm{m}, 1.14 \mu \mathrm{m}$ and $1.4 \mu \mathrm{m}$ are observed for the 2-bar $\mathrm{CO}_{2}$ case. This enhanced absorption is the result of increased $\mathrm{H}_{2} \mathrm{O}$ abundances for the 2-bar $\mathrm{CO}_{2}$ case, as compared to the two lower- $\mathrm{CO}_{2}$ cases (Fig. 1), due to the higher surface temperature. The model tropospheres assume a fixed distribution a)

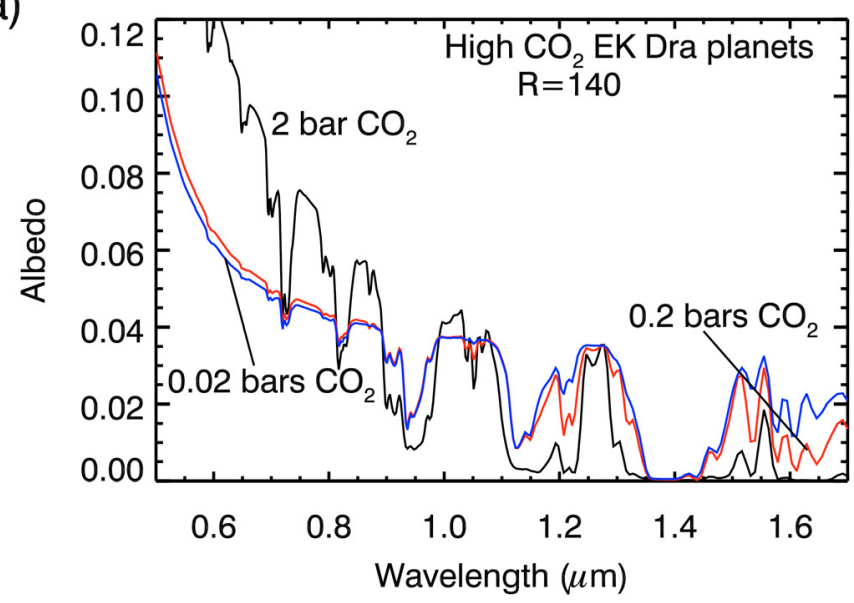

b)

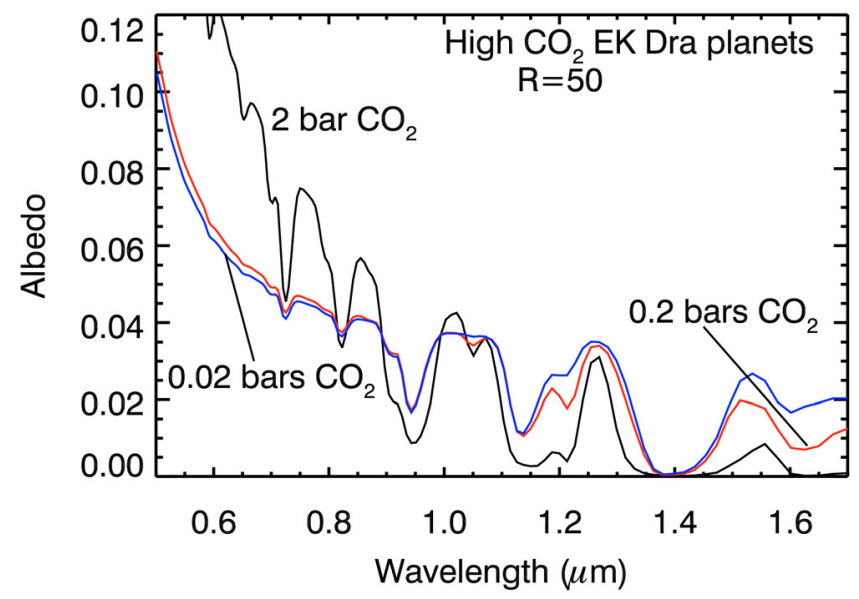

Fig. 10. Spectra for the $0.02,0.2$ and 2-bar $\mathrm{CO}_{2}$ cases convolved with a triangular slit function and degraded to a constant wavenumber resolution equivalent to a) $R \sim 140$, and b) $R \sim 50$.

of relative humidity; hence, $\mathrm{H}_{2} \mathrm{O}$ abundances increase exponentially with increasing surface temperature, following the corresponding increase in the saturation vapor pressure. The water absorption features can be identified, and different concentrations discriminated between, at resolutions as low as $R \sim 20$. However the ability to separate other species from the water vapor (such as the $1.2 \mu \mathrm{m} \mathrm{CO}_{2}$ absorption feature) at $R \sim 20$ becomes extremely difficult, instead requiring resolutions closer to $R \sim 50-70$ to do so, even for high $\mathrm{S} / \mathrm{N}$.

In the MIR, the spectra are dominated again by absorption from $\mathrm{CO}_{2}$, and these early Earth spectra are not at all similar to that of modern Earth (Fig. 11a). In particular, for the 2-bar $\mathrm{CO}_{2}$ case, the spectrum is heavily distorted by the $\mathrm{CO}_{2}$ absorption, which narrows the Earth's normal atmospheric window region between $8-13 \mu \mathrm{m}$ down to only $8-9 \mu \mathrm{m}$. The effects of collisionally-induced absorption are more pronounced here for the 2-bar $\mathrm{CO}_{2}$ case, and manifest themselves in this wavelength range primarily as a broadening of the $\mathrm{CO}_{2}$ isotope bands, enhancing the wing absorption near 7.4 and $8.0 \mu \mathrm{m}$. While there is some theoretical justification for $\mathrm{CO}_{2}$ pressure-induced continuum absorption within the $10 \mu \mathrm{m}$ and $15 \mu \mathrm{m}$ bands, existing measurements show that this absorption is apparently very weak compared to the allowed transitions at these wavelengths. We are not aware of any quantitative estimates of its intensity. For 
a)
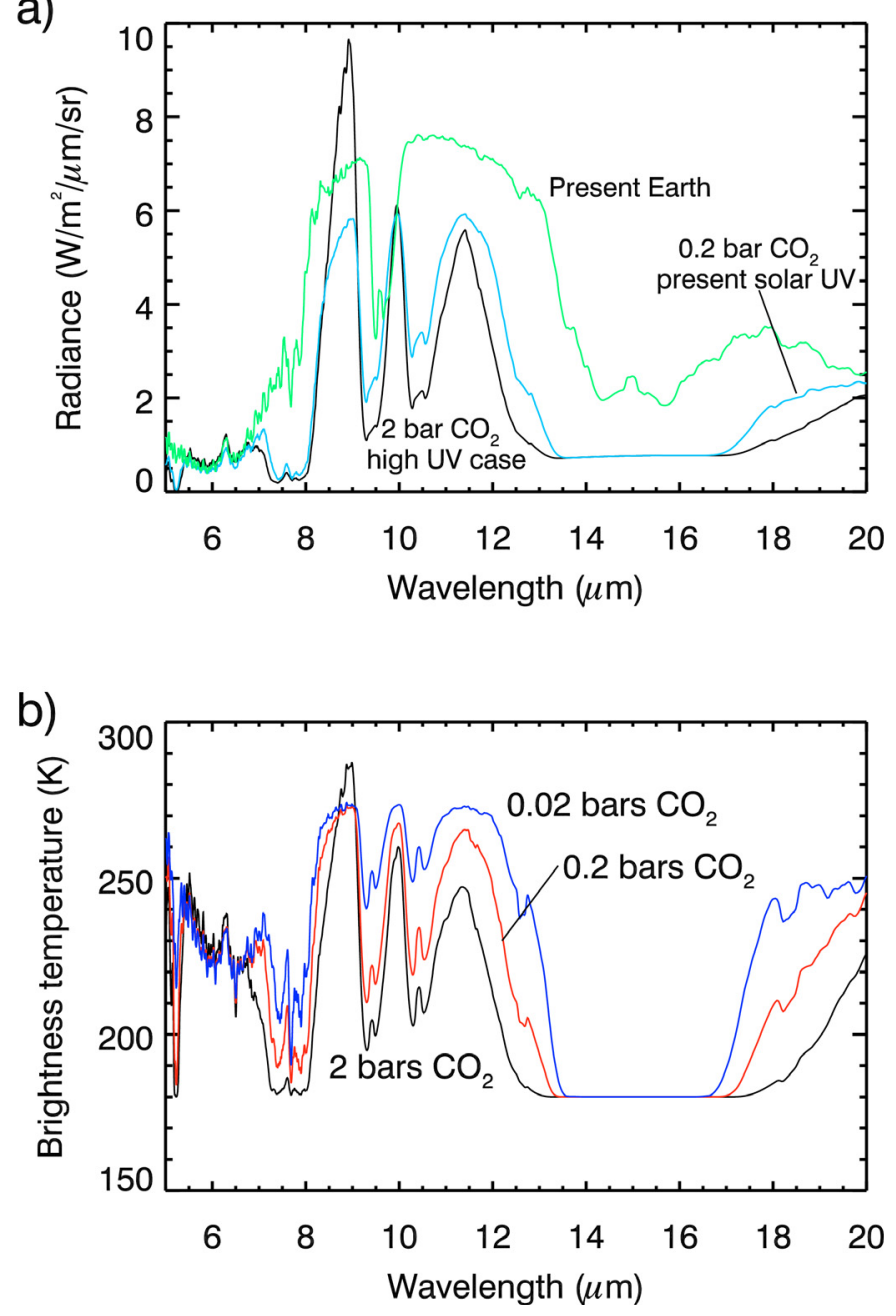

Fig. 11. a) MIR spectra for present Earth (green), our standard case (blue, $0.2 \mathrm{CO}_{2}$ bar, present solar UV) and 2 bars (black) of $\mathrm{CO}_{2}$ around EK Dra. b) MIR spectra of planets with 0.02 (dark blue), 0.2 (red) and 2 bars (black) of $\mathrm{CO}_{2}$ around EK Dra.

example, recent high-pressure (200 atmosphere) measurements by Hartmann's group (cf. Niro et al. 2004) show $\sim 10 \%$ residuals with respect to their best line mixing model in simulations of absorption at pressures at 200 atmospheres. Some of these residuals might be associated with collision-induced absorption, which is neglected in their model. In any case, if this absorption scales as $p^{2}$, it should be $10^{4}$ times weaker for a 2-bar atmosphere, and would therefore have a negligible effect on the modeled spectrum within the context of the work reported here.

In all three cases, methane absorption at $7.7 \mu \mathrm{m}$ is greatly enhanced over that of modern Earth, given that higher concentrations of this compound are present in these low- $\mathrm{O}_{2}$ cases (Table 2). Looking at the MIR brightness temperatures for these three cases (Fig. 11b), the atmospheric window in the 2-bar $\mathrm{CO}_{2}$ case exhibits an enhanced brightness temperature of $287 \mathrm{~K}$ when compared with the $272 \mathrm{~K}$ brightness temperature for the other two cases. This is due to the fact that the atmosphere is considerably hotter overall, but note that the surface temperature for the 2-bar $\mathrm{CO}_{2}$ case is $317 \mathrm{~K}$, and for the other two, $278 \mathrm{~K}$. In all cases, even though no clouds were present, it was not possible to retrieve the surface temperature of the planet by measuring the brightness temperature in the atmospheric window. This is due to the fact that the atmospheric window is not completely

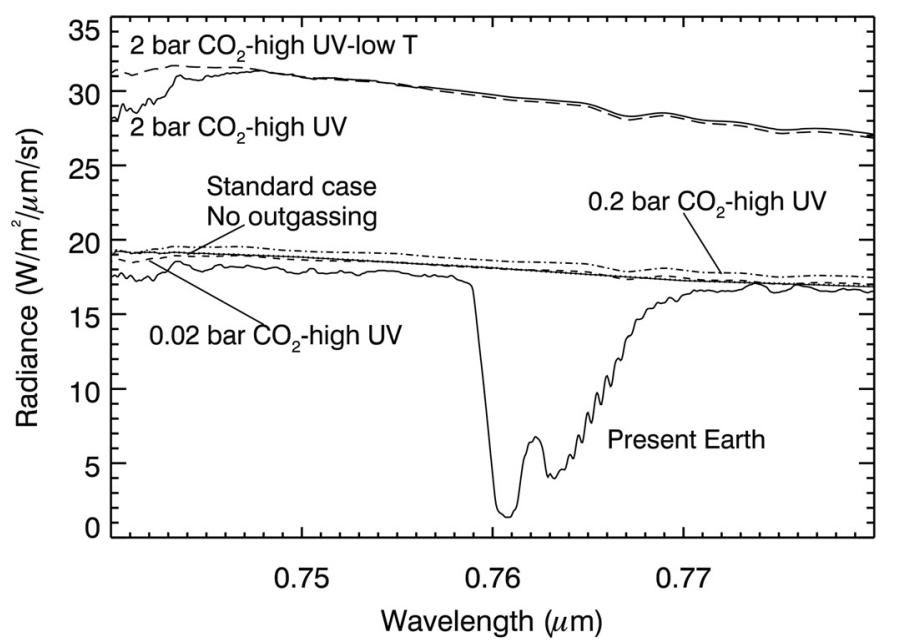

Fig. 12. $\mathrm{O}_{2}$ A-band for all the simulated atmospheres.

transparent, but includes weak absorption from water vapor in an Earth-like atmosphere, and potentially other absorbers in a non-Earth-like atmosphere. The water vapor, which is much enhanced in the 2-bar $\mathrm{CO}_{2}$ case has effectively truncated the atmospheric column at a level higher and cooler than the surface. For the 2-bar atmosphere the $30 \mathrm{~K}$ discrepancy between the actual surface and the measured brightness temperatures corresponds to a sampled altitude of $\sim 4 \mathrm{~km}$ above the surface.

Direct comparison between spectra generated for the 0.2 bar $\mathrm{CO}_{2}$ planetary atmospheres for high-UV (EK Dra) and low-UV (Sun) cases show very few differences, except for changes in the strength of the methane absorption features for both the vis-NIR and MIR. This is due to the UV-induced removal of $\mathrm{CH}_{4}$ from the EK Dra planet, which reduces the overall $\mathrm{CH}_{4}$ mixing ratio by a factor of $\sim 3$ for the high-UV case, as can be seen in Table 2 .

Finally, the modeled strength of the $\mathrm{O}_{2}$ A-band feature in the visible, for all the planetary cases considered here (Fig. 12) demonstrates that the generation of a detectable false positive for oxygen is highly unlikely, even with very high spectroscopic resolution and $\mathrm{S} / \mathrm{N}$.

\section{Discussion}

Our results show that for a habitable planet, rainout of oxidized species onto a reduced surface will inhibit the generation of an abiotic "false positive" signal with strong $\mathrm{O}_{2}$ or $\mathrm{O}_{3}$ absorption. As summarized in Fig. 12, this result is robust to both high stellar UV output and high atmospheric $\mathrm{CO}_{2}$ abundances, as abiotic $\mathrm{O}_{2}$ and $\mathrm{O}_{3}$ are not visible for any of our cases, even in the extreme case of a planet with an atmosphere containing 2-bars of $\mathrm{CO}_{2}$ and in orbit around a high-UV star. We also explored the sensitivity of atmospheric constituents and their detectability to volcanic outgassing rates, incident UV flux, and atmospheric composition. We found that the most significant spectral effects of either turning off volcanic outgassing, or increasing UV flux, was to noticeably decrease the observed methane absorption. Increased stellar UV flux had little observable effect on the rest of the planetary spectrum, even though modest enhancements were seen in the atmospheric $\mathrm{O}_{3}$ and $\mathrm{O}_{2}$ column depths.

Our results contradict previous models (Selsis et al. 2002) that predicted high abiotic abundances of $\mathrm{O}_{2}$ and $\mathrm{O}_{3}$ in planets with $\mathrm{CO}_{2}$-rich atmospheres. As explained earlier, these models failed to balance the $\mathrm{H}_{2}$ budget because they ignored the rainout of soluble oxidized and reduced compounds. Including these 
terms-particularly the rainout of oxidants such as $\mathrm{H}_{2} \mathrm{O}_{2}, \mathrm{H}_{2} \mathrm{SO}_{4}$, and $\mathrm{HNO}$, ensures that an abiotic atmosphere on an Earth-like planet should contain substantial concentrations of $\mathrm{H}_{2}$ and only minimal amounts of $\mathrm{O}_{2}$ and $\mathrm{O}_{3}$.

That said, there still may be planets within the habitable zones of their parent stars that do not have active hydrological cycle for which calculations similar to those of Selsis et al. may be valid and on which abiotic $\mathrm{O}_{2}$ and $\mathrm{O}_{3}$ could conceivably build up. For example, if a planet in the habitable zone lacked significant liquid water, perhaps because it formed with no appreciable water inventory, as has been modeled for terrestrials in planetary systems with eccentric Jovians (Raymond et al. 2004), then our entire analysis of the hydrogen budget would be invalid. $\mathrm{H}_{2}$ would not be produced by reaction of photochemically produced oxidants with reduced species in the crust and ocean, nor would it be likely to be outgassed from volcanoes. This would be compensated by a complete lack of hydrogen escape to space, thereby eliminating the net source of $\mathrm{O}_{2}$ identified earlier in this paper. Some abiotic $\mathrm{O}_{2}$ would presumably accumulate in the atmosphere due to photolysis of $\mathrm{CO}_{2}$, although we have not attempted to quantify $\mathrm{O}_{2}$ production for dessicated planets in this paper. However, abiotic production of $\mathrm{O}_{2}$ on such a water-free planet could be relatively easy identified by the complete lack of $\mathrm{H}_{2} \mathrm{O}$ bands in the planet's visible and MIR spectrum, which would indicate that it was unlikely to be an abode for Earth-like, water-and-carbon-based life.

Another exception to our analysis would be a frozen, "Snowball Earth" type planet within the habitable zone of its star. Earth itself appears to have undergone global glaciation at least 3 times during its history, most recently in the Neoproterozoic, at $2610 \mathrm{Myr}$ and $720 \mathrm{Myr}$ ago (Hoffman et al. 1998). On such a frozen planet, as on the dry planet described above, photochemically produced oxidants would not react with reduced materials in the planet's crust and ocean. The planet would thus be similar to the Mars-like planet mentioned in the introduction for which atmospheric $\mathrm{O}_{2}$ could, in theory, continue to accumulate leading to a possible "false positive" for life.

Two things mitigate against this posing a significant problem for life detection by TPF or Darwin. One is that Snowball Earth episodes on Earth are thought to be relatively short-lived, 10's of millions of years or less, because they are eventually terminated by buildup of volcanic $\mathrm{CO}_{2}$ in the atmosphere (Hoffman et al. 1998). $10 \mathrm{Myr}$ represents only about $0.2 \%$ of the Earth's geologic history, and the odds of finding an Earth-like planet in such a state in our Solar neighborhood are relatively small. Second, it should be possible to identify whether a planet is or not in a Snowball Earth-type phase, either by looking for the presence of water ice or water vapor bands in their spectrum, or by follow-up measurements of polarization to characterize the planetary surface and search for the presence of liquid water.

To quantify the effect of decreasing global surface temperature on the detectability of water vapor we ran a series of models similar to our standard, 0.2 bar $\mathrm{CO}_{2}$ case, but for successively lower surface temperatures. The corresponding synthetic spectra generated from these environments are shown in Figs. 13 and 14. Model calculations of Snowball Earth (Hyde et al. 2000) show that the surface temperature drops precipitously from $\sim 275 \mathrm{~K}$ to $230 \mathrm{~K}$ when global glaciation sets in. For an atmosphere with Earth's relative humidity distribution, Fig. 13 shows that the water vapor bands in the visible and near-IR become extremely weak as this transition occurs with a $238 \mathrm{~K}$ surface temperature planet showing only $\sim 5 \%$ of the absorption strength in the $1.13 \mu \mathrm{m}$ water band when compared to the feature seen for the $278 \mathrm{~K}$ surface temperature planet. However, with high $\mathrm{S} / \mathrm{N}$ and

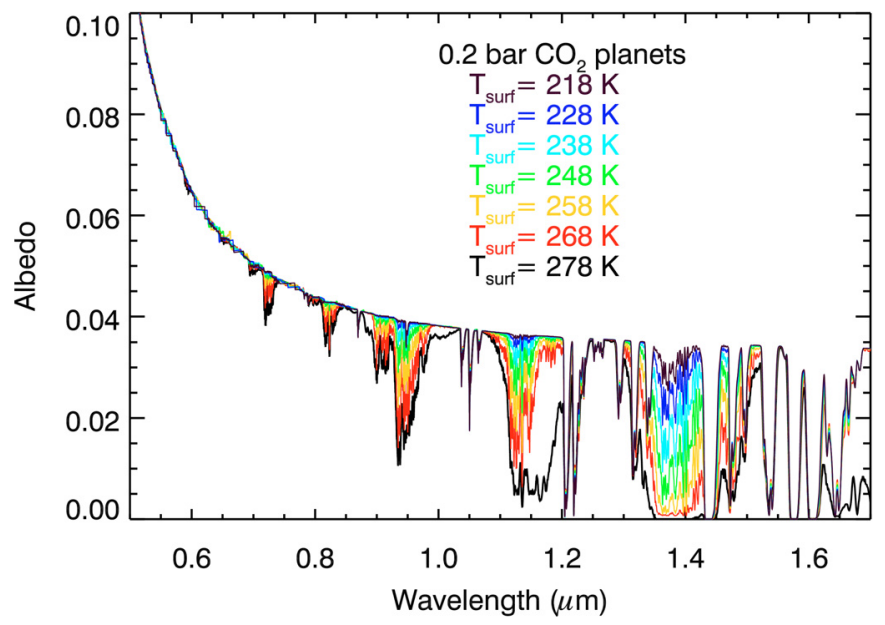

Fig. 13. Albedo of planets with 0.2 bars of $\mathrm{CO}_{2}$ around the Sun with different surface temperatures.
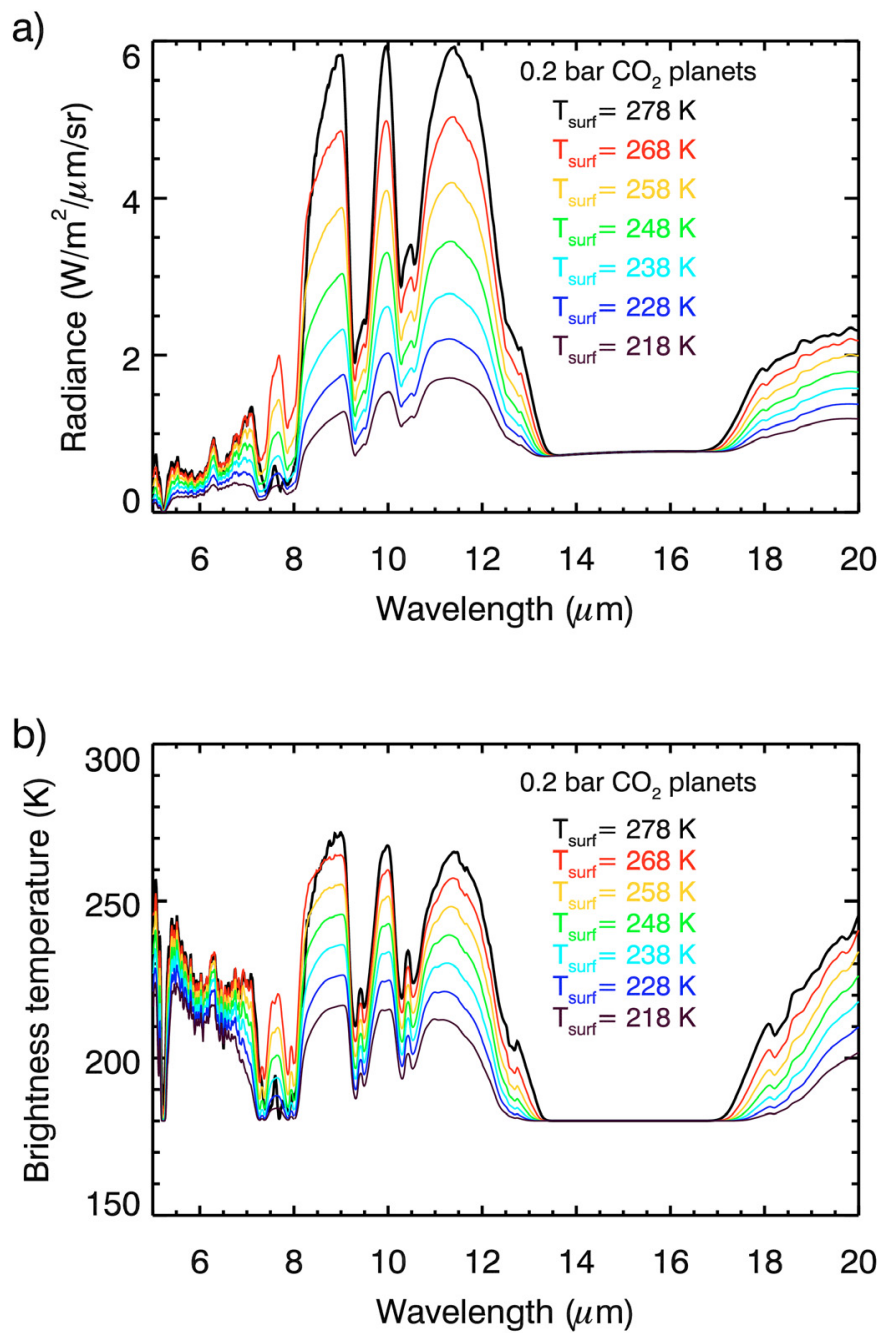

Fig. 14. MIR spectra of planets with 0.2 bars of $\mathrm{CO}_{2}$ around the Sun with different surface temperatures. a) Radiance b) brightness temperature.

a spectral resolution greater than $R \sim 50$, the $1.13 \mu$ m feature could be detected at temperatures greater than $238 \mathrm{~K}$. It must be stressed that $\mathrm{S} / \mathrm{N}$ and resolution are trade offs, as lower resolutions can require higher $\mathrm{S} / \mathrm{N}$ to detect a given feature. In the MIR (Fig. 14) the changes in the spectrum with decreasing surface temperature are seen as an interplay between lower water 
vapor amounts and the atmospheric temperature. The declining planetary atmospheric temperatures with surface temperature are seen in these cloud-free cases in the atmospheric window region between $8-13 \mu \mathrm{m}$. Although note that even in these cloud-free cases, the peak temperature detected in the atmospheric window at $9 \mu \mathrm{m}$ is not the surface temperature, being several degrees cooler due to the presence of water vapor, which results in atmospheric temperatures sampled at levels higher than the surface (Fig. 14b). If clouds were present, the inferred temperature would typically be even colder. The declining water vapor amounts are seen in the difference in the brightness temperature between the 5.5-7.0 $\mu \mathrm{m}$ water vapor region and the $8-13 \mu \mathrm{m}$ window region. Correct interpretation of these spectra, however, would require generation of synthetic spectra that could be used to disentangle the combined effects of water and temperature on the spectrum. Nonetheless, as was the case more straightforwardly for the visible-NIR spectrum, it may be possible to detect the significantly lower water vapor amount, given instrumentation that was sensitive to the $6.3 \mu \mathrm{m}$ water vapor band. In this case, a resolution greater than $R \sim 20$ in the MIR might be sufficient, with high $\mathrm{S} / \mathrm{N}$. However, we add the caveat that the presence of clouds, either planet-wide, or covering a smaller fraction of the visible planetary disk, could skew the interpretation to colder temperatures and lower water abundances than are present at the planet's surface. For these cases, searching for abundant water-soluble atmospheric gases, such as $\mathrm{SO}_{2}$ might serve as a secondary indicator that we are observing a planet without a surface ocean, rather than one with clouds obscuration. Multiple samples of the planet's atmosphere over time may also help to determine whether clouds are affecting the observed temperatures and water abundances.

Another way to test for the presence of liquid water on an exoplanet's surface is to measure the polarization of light reflected from its surface. The specular "glint" produced by the smooth ocean surface should be strongly polarized, at least at some phase angles (McCullough 2007; Stam et al. 2006; West et al. 2005; Williams \& Gaidos 2004). Separation of different polarizations is a feature of some suggested techniques for coronagraphic starlight suppression. Even if this measurement was not performed by an initial TPF-C mission, it could presumably be done by some follow-up mission as a way of verifying the presence of surface liquid water (or at least of some surface liquid). A positive detection of either $\mathrm{O}_{2}$ or $\mathrm{O}_{3}$ in an exoplanet's atmosphere would certainly warrant further investigation to be sure that the liquid water required for life was present at the planet's surface.

\subsection{Applicability to other planetary systems}

For these simulations, we used EK Dra, a young, UV-active star with a calculated age of only $10^{8}$ years. If we were considering only whether this star was likely to harbor habitable planets, then this would be a poor choice, as planets around a star of this age would still be undergoing large, ocean-vaporizing impacts (Sleep et al. 1989). We would not anticipate seeing planetwide biosignatures at this early and hostile stage, and so would also be unlikely to mistake a false positive as a biosignature. Nonetheless, EK Dra is an excellent example for our simulations, as it most realistically represents the approximate upper limit on the UV radiation likely to be encountered by a planet around a Sun-like star.

When the first studies of the UV environment for early Earth were published, the only known examples of young Sun-like stars were the T Tauri type stars. IUE observations showed that
$\mathrm{T}$ Tauri UV fluxes were as much as $10^{4}$ times higher than the present solar UV radiation. Based on those observations and stellar evolution models, those early studies concluded that the UV enhancement for a $10^{8}$ year old, Sun-like star, should be of the order of 10 times the present solar UV flux (Canuto et al. 1982; Zanhle \& Walker 1982). However, we believe that this is an overestimate. EK Dra, at $10^{8}$ years, and one of the most UV active stars known (Ribas et al. 2005), does exhibit fluxes 10 times that of the Sun at wavelengths shortward of $160 \mathrm{~nm}$. However, when this flux is integrated over the wavelength range most relevant to atmospheric chemistry $(\lambda<200 \mathrm{~nm})$, it is only $\sim 4.5$ times larger than that of the Sun, on average. More importantly for the atmospheric chemistry, the continuum photon flux (rather than the energy flux) at $\lambda<200 \mathrm{~nm}$ is just $\sim 3.6$ times higher in EK Dra when compared to the solar spectrum (cf. Fig. 3) (Note that our continuum calculations do not include the Ly $\alpha$ line which we calculated to be $\sim 10$ times larger than for the present Sun, and which was included in all our modeling simulations). These considerations suggest that our results can be interpreted as an upper limit on detectability for high- $\mathrm{CO}_{2}$ atmospheres around young, UV-active stars that are either Sun-like, or cooler than our Sun. If we consider stars with higher-UV flux than our Sun, e.g. the F stars, it is also highly likely that our results are applicable. An F2V star has an integrated UV flux that is approximately 2.5 times that of EK Dra (Segura et al. 2003). An examination of our Table 2 indicated that the increase in $\mathrm{O}_{2}$ and $\mathrm{O}_{3}$ column depth in a high- $\mathrm{CO}_{2}$ atmosphere as a result of EK Dra's 4.5 times higher flux is relatively modest, being 3.6 and 2.9 times respectively, and this is for column depth values that are still $10^{3}$ times lower than those likely to be detectable for a planet around an F2V star (see Segura et al. 2003, Table 1 and Fig. 14). It is therefore unlikely that the additional increase of 2.5 in the UV flux from an F star would result in detectable $\mathrm{O}_{2}$ and $\mathrm{O}_{3}$ for the high- $\mathrm{CO}_{2}$ planets modeled here. We therefore conclude that the results presented here are an upper limit for the majority of parent stars likely to be considered as TPF/Darwin targets.

\subsection{Effect of clouds on our conclusions}

All the simulations shown here were run for clear-sky atmospheres, with no appreciable hazes or cloud layers, and isothermal stratospheres. These cases shown here are therefore the most sensitive for detecting $\mathrm{O}_{2}$ and $\mathrm{O}_{3}$ in our planetary spectrum, as they allow a longer pathlength through the atmosphere that is not truncated by clouds. Additionally, the contrast between the hot surface and lower atmosphere, and the cool, isothermal stratosphere provides for maximum detectability in the mid-infrared. However, given that both the $\mathrm{O}_{3}$ and $\mathrm{O}_{2}$ concentrations peak above the tropopause in all our model cases, and have significantly smaller abundances below that, a cloud at the tropopause would not significantly change the net column abundance of $\mathrm{O}_{2}$ and $\mathrm{O}_{3}$, and hence their detectability. In the case of the $\mathrm{CO}_{2}$ however, which is evenly mixed through our atmospheres, a cloud at the tropopause would significantly truncate the observed column, and would result in much reduced absorption depths for $\mathrm{CO}_{2}$ in the visible, and would also dramatically reduce the $\mathrm{CO}_{2}$ absorption in the mid-infrared, as the cooler clouds tops would obscure the hotter surface and lower atmosphere and provide a reduced temperature difference with the stratosphere. The brightness temperatures of the observed emitting layer would also be reduced to that of the cloud top temperatures, and would preclude our ability to determine the planet's surface temperature. This case can be demonstrated by comparing the brightness 


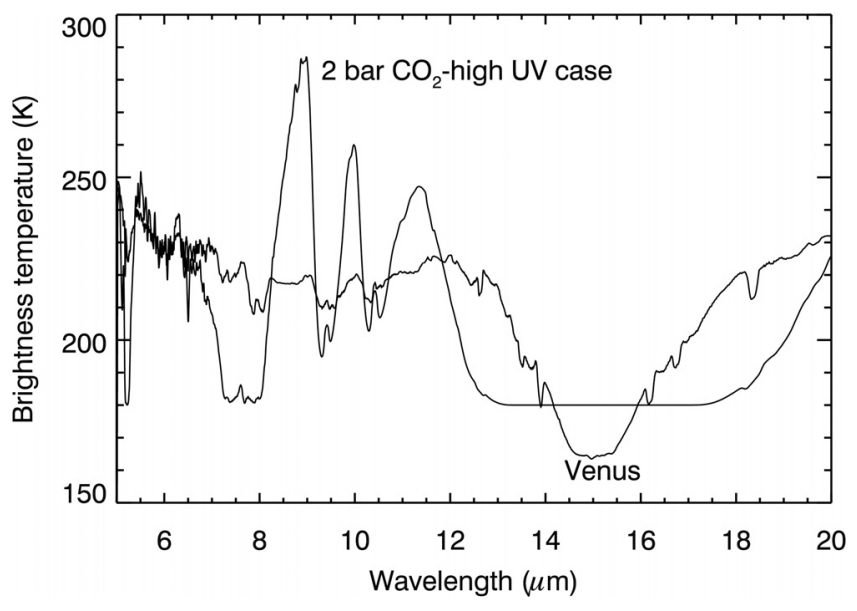

Fig. 15. Spectra of the 2 bars of $\mathrm{CO}_{2}$ planet around EK Dra and Venus.

temperatures for the 2-bar $\mathrm{CO}_{2}$ atmosphere and a modeled spectrum of the planet Venus (Fig. 15). Even though Venus is known to have a 93 bar $\mathrm{CO}_{2}$ atmosphere and a $730 \mathrm{~K}$ surface temperature, its $\mathrm{CO}_{2}$ absorption is significantly weaker than seen in our cases, and its maximum brightness temperature in the MIR is only $250 \mathrm{~K}$, due to its planet-wide cloud deck.

\subsection{Implications for extrasolar planet characterization missions}

TPF-C, TPF-I, and Darwin will all have the opportunity to study a wide variety of planetary systems. If terrestrial planets are found around these stars, it is highly likely that we will see the atmospheres of planets, like our own Solar System examples of Venus, Mars and the early Earth, that are dominated by $\mathrm{CO}_{2}$. Therefore, understanding the appearance of $\mathrm{CO}_{2}-$ dominated atmospheres, and their ability to generate false positives is an important step in characterizing extrasolar terrestrial planets that are not strictly Earth-like. In addition to disproving the likelihood of significant abiotic sources of $\mathrm{O}_{2}$ and $\mathrm{O}_{3}$ in a $\mathrm{CO}_{2}$-dominated atmosphere, we have also demonstrated that $\mathrm{CO}_{2}$-dominated habitable atmospheres exhibit a wealth of spectral features. The multiple $\mathrm{CO}_{2}$ absorption bands throughout the visible-NIR range accessible to an optical coronograph $(0.5-$ $1.7 \mu \mathrm{m})$ and an MIR interferometer $(5-25 \mu \mathrm{m})$ provide differing sensitivity to a large range of $\mathrm{CO}_{2}$ abundances. The most sensitive band in the visible-NIR is near $1.62 \mu \mathrm{m}$, and produces a potentially detectable feature, even for the Earth's present $330 \mathrm{ppm}$ abundance. For atmospheres with higher $\mathrm{CO}_{2}$ abundances $(0.2-$ 2 bars of $\mathrm{CO}_{2}$ ), the less sensitive $1.05 \mu \mathrm{m}$ bands, which are weak in the Earth's atmosphere, provide strong absorption features, and are better features for potential quantification of the planet's atmospheric $\mathrm{CO}_{2}$ provided the spectral resolution is above 50 . In the MIR, the $\mathrm{CO}_{2} 15 \mu \mathrm{m}$ feature provides the strongest absorption for $\mathrm{CO}_{2}$ and is the most detectable feature in a terrestrial planet atmospheres. In addition, we have shown that in the MIR the isotopes of $\mathrm{CO}_{2}$, produce strong features. This is arguably one of the most detectable isotope features in a terrestrial planet spectrum, even at relatively poor spectral resolution $(R \sim 20)$ and signal to noise. These features may allow us to get a first measure of $\mathrm{O}$ isotopic composition of an early-Earth-like or $\mathrm{CO}_{2}$-dominated terrestrial planet. As lighter isotopes tend to evaporate more readily, and heavier isotopes are rained out more efficiently, the atmospheric $\mathrm{O}$ isotope inventory may in turn provide secondary clues to the presence and degree of activity in a planet's hydrological cycle.

\section{Conclusions}

Our results show that for a habitable planet with abundant liquid water, rainout of oxidized species onto a reduced surface will keep atmospheric $\mathrm{H}_{2}$ sufficiently high, that the generation of an abiotic "false positive" signal with strong $\mathrm{O}_{2}$ or $\mathrm{O}_{3}$ absorption is unlikely, and does not pose a significant hazard for habitable planets observed with TPF or Darwin. This result is robust to realistic limits on the UV output of the star, and the abundance of $\mathrm{CO}_{2}$ in the planet's atmosphere. Dense $\mathrm{CO}_{2}$ atmospheres of rocky planets may also display a range of distinctive spectral features in both the visible and MIR that allow us to at least qualitatively determine the prevalence of $\mathrm{CO}_{2}$ in the atmosphere, and potentially measure atmospheric isotopic ratios for oxygen.

Acknowledgements. This material is based upon work performed by the NASA Astrobiology Institute's Virtual Planetary Laboratory Lead Team, supported by the National Aeronautics and Space Administration through the NASA Astrobiology Institute under Cooperative Agreement No. CAN-00-OSS-01. J.F.K. also acknowledges support from NASA's Exobiology and Astrobiology Programs. M.C. thanks NASA for supporting his participation in this work under grant NNG04GL49G with UC Berkeley. We thank Patrick Kasting for the computational support provided. Review by F. Selsis greatly improved the manuscript and is acknowledged with gratitude.

\section{References}

Angel, J. R. P., Cheng, A. Y. S., \& Woolf, N. J. 1986, Nature, 322, 341

Berkner, L. V., \& Marshall, L. L. 1964, Disc. Faraday Soc., 34, 122

Berkner, L. V., \& Marshall, L. L. 1965, J. Atmos. Sci., 22, 225

Berkner, L. V., \& Marshall, L. L. 1966, J. Atmos. Sci., 23, 133

Berkner, L. V., \& Marshall, L. L. 1967, Adv. Geophys., 12, 309

Brinkman, R. T. 1969, J. Geophys. Res., 74, 5355

Canuto, V. M., Levine, J., Augustsson, T., \& Imhoff, C. 1982, Nature, 296, 816

Canuto, V. M., Levine, J. S., Augustsson, T. T., Imhoff, C. L., \& Giampapa, M. S. 1983 Nature, 305, 281

Cloud, P. E. 1972, Amer. J. Sci., 272, 537

Clough, S. A., Kneizys, F. X., \& Davies, R. W. 1989, Atmos. Res., 23, 229

Cockell, C. S., \& Horneck, G. 2001, Photochem. Photobiol. 73, 447

Cohen, M., Megeath, S. T., Hammersley, P. L., Martín-Luis, F., \& Stauffer, J. 2003, AJ, 125, 2645

Crisp, D. 1997, Geophys. Res. Lett., 24, 571

Des Marais, D. J. et al. 2002, Astrobiology, 2, 153

Dorren, J. D., \& Guinan, E. F. 1994, ApJ, 428, 805

Emerich, C., Lemaire, P., Vial, J.-C. et al. 2005, Icarus, 178, 429

Giorgi, F., \& Chameides, W. L. 1985, J. Geophys. Res., 90, 7872

Goody, R. M., \& Yung, Y. L. 1989, Atmospheric radiation: theoretical basis (New York: Oxford University Press).

Gray, R. O., Corbally, C. J., Garrison, R. F., McFadden, M. T., \& Robinson, P. E. 2003, AJ, 126, 2048

Gruszka, M., \& Borysow, A. 1996, Mol. Phys., 88, 1173

Gruszka, M., \& Borysow, A. 1997, Icarus, 129, 172

Gruszka, M., \& Borysow, A. 1998, Mol. Phys., 93, 1007

Hartmann, J.-M., \& Boulet, C. 1991, J. Chem. Phys., 94, 6406

Hoffman, P. F., Kaufman A. J., Halverson, G. P \& Schrag, D. P. 1998, Science, 281,1342

Holland, H. D. 1978, The Chemistry of the Atmosphere and Oceans (New York: Wiley)

Holland, H. D. 1984, The Chemical Evolution of the Atmosphere and Oceans (Princeton: Princeton University Press)

Holland, H. D. 2002, Geochim. Cosmochim. Acta, 66, 3811

Houghton, J. T., Meira Filho, L. G., Callander, B. A., et al. 1995, Climate Change 1994: Radiative Forcing of Climate Change and an Evaluation of the IPCC IS92 Emission Scenarios (Cambridge, MA: Cambridge University Press)

Hunten, D. M. 1973, J. Atmos. Sci., 30, 1481

Hyde, W. T., Crowley T. J., Baum, S. K., \& Peltier, W. R. 2000, Nature, 405, 425 Kasting, J. F. 1990, Origins Life Evol. Bios., 20, 199

Kasting, J. F. 1993, Science, 259, 920

Kasting, J. F. 1997, Origins Life, 27, 291

Kasting, J. F., \& Brown, L. L. 1998, Setting the stage: the early atmosphere as a source of biogenic compounds, Brack, A. The Molecular Origins of Life: Assembling the Pieces of the Puzzle (New York: Cambridge Univ. Press), 35

Kasting, J. F., \& Catling, D. C. 2003, ARA\&A, 41, 429 
Kasting, J. F. \& Donahue, T. M. 1980, J. Geophys. Res., 85, 3255

Kasting, J. F., Liu, S. C., \& Donahue, T. M. 1979, J. Geophys. Res., 84, 3097

Kasting, J. F., Pollack J. B., \& Ackerman T. P. 1984a, Icarus, 57, 335

Kasting, J. F., Pollack, J. B., \& Crisp, D. 1984b, J. Atmos. Chem, 1, 403

Kasting, J. F., Whitmire, D. P., \& Reynolds, R. T. 1993, Icarus, 101, 108

Kelley, D. S., Karson, J. A., Früh-Green, G. L., et al. 2005, Science, 307, 1428

Kharecha, P. Kasting, J. F., \& Siefert, J. L. 2005, Geobiol., 3, 53

Kurucz, R. L. 1993, CD-ROM 13, ATLAS9 Stellar Atmosphere Programs and

$2 \mathrm{~km} \mathrm{~s}^{-1}$ Grid (Cambridge: Smithsonian Astrophys. Obs.)

Lee, Y. N., \& Schwartz, S. E. 1981, J. Geophys. Res., 86, 11971

Leger, A., Pirre, M., \& Marceau, F. J. 1993, A\&A, 277, 309

Levine, J. S., Hays, P. B., \& Walker, J. C. G. 1979, Icarus, 39, 295

Liang, M. C., Hartman, H., Kopp, R. E., Kirschvink, J. L., \& Yung, Y. L. 2006, Proc. Nat. Acad. Sci., 103, 896

Massa, D., \& Fitzpatrick, E. L. 2000, ApJS, 126, 517

Massie, S. T., \& Hunten, D. M. 1981, J. Geophys. Res., 86, 9859

McElroy, M. B., \& Donahue, T. M. 1972, Science, 177, 986

McClatchey, R. A., Fenn, R. W., Selby, J. E. A., Volz, F. E., \& Garing, J. S. 1971, Technical Report ASCRL-71-0279 (Bedford, MA: Air Force Cambridge Research Laboratory)

McCullough, P. R. 2007, ApJ, submitted

Meadows, V. S., \& Crisp, D. 1996, J. Geophys. Res., 101, 4595

Montes, D., López-Santiago, J., Fernández-Figueroa, M. J., \& Gálvez, M. C. 2001, A\&A, 379, 976

Moore, J. F., 1971, Ph.D. Thesis, Columbia University, 31

Niro, F., Boulet C., \& Hartman, J.-M. 2004, J. Quan. Spectros. Rad. Trans., 88, 483
Owen, T. 1980, Strategies for Search for Life in the Universe, ed. M. D. Papagiannis (Dordrecht: Reidel), 177

Pavlov, A. A., Brown, L. L., \& Kasting, J. F. 2001, J. Geophys. Res., 106, 23267

Pavlov, A. A., Kasting, J. F., Brown, L. L., Rages, K. A., \& Freedman, R. 2000, J. Geophys. Res., 105, 11981

Raymond, S. N., Quinn, T., \& Lunine, J. I. 2004, Icarus, 168, 1

Ribas, I., Guinan, E. F., Güdel, M., \& Audard, M. 2005, ApJ, 622, 680

Rosenkranz, P. W. 1988, J. Quant. Spectros. Rad. Trans., 39, 287

Schindler, T. L., \& Kasting, J. F. 2000, Icarus, 145, 262

Segura, A., Krelove, K., Kasting, J. F., et al. 2003, Astrobiol., 3, 689

Selsis, F., Despois, D., \& Parisot, J.-P. 2002, A\&A, 388, 985

Sleep, N. H., Zahnle, K. J., Kasting, J. F., \& Morowitz, H. 1989, Nature, 342, 139

Sleep, N., \& Bird, D.K. 2007, Geobiology, 5, 101

Slinn, W. G. N., et al. 1978, Atmos. Environ., 12, 2055

Stam, D. M., de Rooij, W. A., Cornet, G., \& Hovenier, J. W. 2006, A\&A, 452, 669

Strassmeier, K. G., \& Rice, J. B. 1998, A\&A, 330, 685

Tian, F., Toon, O. B., Pavlov, A. A., \& De Sterck, H. 2005, Science, 308, 1014

Walker, J. C. 1977, Evolution of the Atmosphere (New York: Macmillan)

West, R. A., Brown, M. E., Salinas, S. V., Bouchez, A. H., \& Roe, H. G. 2005, Nature, 436, 670

Williams, D. M., \& Gaidos E. 2004, Bull. Am. Astron. Soc., 36, 1173

World Metereological Organization, 1985, Atmospheric Ozone (Washington, DC: National Aeronautics and Space Administration), 1

Zahnle, K. J., \& Walker, J. C. G. 1982, Rev. Geophys. Space Phys., 20, 280 